\title{
Data science in economics: comprehensive review of advanced machine learning and deep learning methods
}

\author{
Saeed Nosratabadi ${ }^{1}$, Amir Mosavi $2,3, *$, Puhong Duan ${ }^{4}$, Pedram Ghamisi ${ }^{5}$, Ferdinand Filip ${ }^{6}$, \\ Shahab S. Band 7,8,*, Uwe Reuter ${ }^{9}$, Joao Gama ${ }^{10}$, Amir H. Gandomi ${ }^{11}$ \\ 1 Doctoral School of Management and Business Administration, Szent Istvan University, Godollo 2100, \\ Hungary; saeed.nosratabadi@phd.uni-szie.hu (S.N.) \\ 2 Bauhaus-Universität Weimar, 99423 Weimar, Germany (A.M.) \\ 3 School of Economics and Business, Norwegian University of Life Sciences, 1430 Ås, Norway \\ 4 College of Electrical and Information Engineering, Hunan University, Changsha 410082, China; \\ puhong_duan@hnu.edu.cn (P.D.) \\ 5 Helmholtz Institute Freiberg for Resource Technology, Helmholtz-Zentrum Dresden-Rossendorf, Dresden, \\ Germany; p.ghamisi@hzdr.de (P.G.) \\ 6 Department of Mathematics, J. Selye University, 94501 Komarno, Slovakia; filipf@ujs.sk (F.F.) \\ 7 Institute of Research and Development, Duy Tan University, Da Nang 550000, Vietnam (S.S.B.) \\ 8 Future Technology Research Center, College of Future, National Yunlin University of Science and \\ Technology 123 University Road, Section 3, Douliou, Yunlin 64002, Taiwan \\ 9 Faculty of Civil Engineering, Technische Universität Dresden, 01069 Dresden, Germany; uwe.reuter@tu- \\ dresden.de (U.R.) \\ 10 Faculty Laboratory of Artificial Intelligence and Decision Support (LIAAD) - INESC TEC, Campus da \\ FEUP, Rua Dr. Roberto Frias, 4200 - 465 Porto, Portugal; jgama@fep.up.pt (J.G.) \\ 11 Faculty of Engineering and Information Technology, University of Technology Sydney, NSW 2007, \\ Australia; gandomi@uts.edu.au (A.H.G.) \\ *Correspondence: amirhosein.mosavi@tdtu.edu.vn; shamshirbandshahaboddin@duytan.edu.vn \\ Received: date; Accepted: date; Published: date
}

\begin{abstract}
This paper provides a state-of-the-art investigation on advances in data science in emerging economic applications. Analysis was performed on novel data science methods in four individual classes of deep learning models, hybrid deep learning models, hybrid machine learning, and ensemble models. Application domains include a wide and diverse range of economics research from the stock market, marketing, and e-commerce to corporate banking and cryptocurrency. Prisma method, a systematic literature review methodology, was used to ensure the quality of the survey. The findings reveal that the trends follow the advancement of hybrid models, which, based on the accuracy metric, outperform other learning algorithms. It is further expected that the trends will converge toward the advancements of sophisticated hybrid deep learning models.
\end{abstract}

Keywords: data science; deep learning; ensemble machine learning models; economics; hybrid machine learning

\section{Introduction}

Due to the remarkable progress in data analysis methods, the use of data science (DS) in various disciplines has been increasing exponentially, especially economics. Literature has shown that advancements of data science in economics have been progressive and have promising results. Several studies suggest that applications of data science in economics can be categorized and studied in various popular technologies, such as deep learning, hybrid learning models, and ensemble algorithms. Machine learning (ML) algorithms provide the ability to learn from data and deliver indepth insight into problems [1]. Researchers use machine learning models to solve various problems associated with economics. Notable applications of data science in economics are presented in Table 
1. Deep Learning (DL), as an emerging field of machine learning, is currently applied in many aspects of today's society from self-driving cars to image recognition, hazard prediction, health informatics, and bioinformatics [2-5]. Several comparative studies have evaluated the performance of DL models with standard ML models, e.g., support vector machine (SVM), K-nearest neighbors (KNN), and generalized regression neural networks (GRNN) in economic applications . The evolution of DS methods has progressed at a fast pace, and every day, many new sectors and disciplines are added to the number of users and beneficiaries of DS algorithms. On the other hand, hybrid machine learning models consist of two or more single algorithms and are used to increase the accuracy of the other models [6,7]. Hybrid models can be formed by combining two predictive machine learning algorithms or a machine learning algorithm and an optimization method to maximize the prediction function [8]. It has been demonstrated that the hybrid machine learning models outperform the single algorithms, and such an approach has improved the prediction accuracy [9-11]. Ensemble machine learning algorithms are one of the supervised learning algorithms that use multiple learning algorithms to improve learning processes and increase predictive accuracy [6]. Ensemble models apply different training algorithms to enhance training and learning from data.

Table 1. Examples of notable classic machine learning methods applied in economics-related fields

\begin{tabular}{|c|c|c|}
\hline Sources & Machine learning models & Objectives \\
\hline Lee et al. [12] & Support Vector Regression (SVR) & Anomaly Detection \\
\hline Husejinović [13] & $\begin{array}{c}\text { Naive Bayesian And C4.5 Decision } \\
\text { Tree Classifiers }\end{array}$ & Credit Card Fraud Detection \\
\hline Zhang [14] & Improved BP Neural Network & $\begin{array}{c}\text { Aquatic Product Export Volume } \\
\text { Prediction }\end{array}$ \\
\hline $\begin{array}{l}\text { Sundar and } \\
\text { Satyanarayana [15] }\end{array}$ & $\begin{array}{c}\text { Multilayer Feed Forward Neural } \\
\text { Network }\end{array}$ & Stock Price Prediction \\
\hline Hew et al. [16] & Artificial Neural Network (ANN) & Mobile Social Commerce \\
\hline $\begin{array}{l}\text { Abdillah and } \\
\text { Suharjito [17] }\end{array}$ & $\begin{array}{c}\text { Adaptive Neuro Fuzzy Inference } \\
\text { System (ANFIS) }\end{array}$ & E-Banking Failure \\
\hline Sabaitytė et al. [18] & Decision Tree (DT) & Customer Behavior \\
\hline $\begin{array}{c}\text { Zatevakhina, } \\
\text { Dedyukhina, and } \\
\text { Klioutchnikov [19] }\end{array}$ & Deep Neural Network (ANN) & Recommender Systems \\
\hline $\begin{array}{l}\text { Benlahbib and } \\
\text { Nfaoui [20] }\end{array}$ & $\begin{array}{c}\text { Naïve Bayes and Linear Support } \\
\text { Vector Machine (LSVM) }\end{array}$ & Sentiment Analysis \\
\hline
\end{tabular}

Various works exist on the state-of-the-art of DS methods in different disciplines, such as image recognition [21], animal behavior [22], renewable energy forecasting [23]. Hybrid methods have also been investigated in various fields, including financial time series [24], solar radiation forecasting [25], and FOREX rate prediction [26], while ensemble methods have been in fields, ranging from breast cancer [27], image categorization[28], electric vehicle user behavior prediction [29], and solar power generation forecasting [30]. Exploration of the scientific databases such as Thomson Reuters Web-of-Science (WoS) shows an exponential rise in using both DL and ML in economics. The results of an inquiry of essential ML and DL in the emerging applications to economics over the past decade is illustrated in Figure 1. Even though many researchers have applied DS methods to address different problems in the field of economics, these studies are scattered. At the same time, no single study provides a comprehensive overview of the contributions of DS in economic-related fields. Therefore, the current study is conducted to bridge this literature gap. In other words, the main objective of this study is to investigate the advancement of DS in three parts: deep learning methods, hybrid deep learning methods, and ensemble machine learning techniques in economics-related fields. The present work answers the following research questions: 1) What economics-related applications have DS contributed to? 2) Which data science models are applied in each of these applications? 


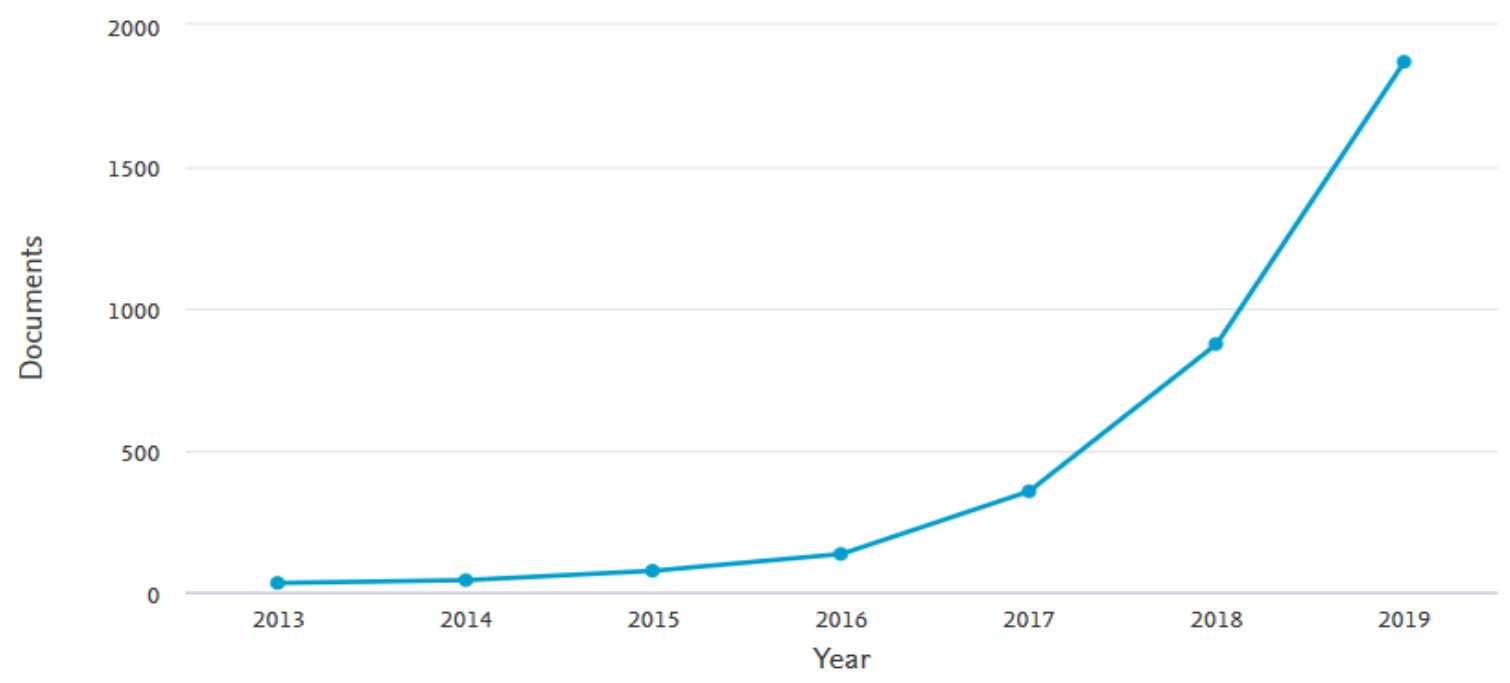

Figure 1. Rapid rise in the applications of data science in economics

The rest of the paper is organized as follows; the method by which the database of this article was formed is described in Section 2. Section 3 presents the findings and discussion, including articles that are categorized by sector and area of study as well as by models. A taxonomy of the application of data science in economics is presented as well. Section 4 discusses the analytical results and the conclusions.

\section{Materials and Methods}

The current study utilized Prisma, a systematic literature review approach, to find the most published articles that have applied data science methods for addressing an issue in a field related to economics. Systematic literature review based on the Prisma method includes four steps: 1) identification, 2) screening, 3) eligibility, and 4) inclusion [31]. In the identification stage, the documents are identified through an initial search among the mentioned databases. In this study, the review was limited to the original peer-review research articles published in Thomson Reuters Webof-Science (WoS) and Elsevier Scopus. Today, the Scopus database includes almost entire authenticated scientific repositories including WoS. Therefore, to avoid overlapping of the inquiries the Scopus has been used as the principal database for search, and the results had been verifies using the WoS repository. This review was limited to articles written in English. This step resulted in 217 articles.

Our inquiry of search included essential machine learning and deep learning methods which used as the search keywords. The search for articles' source included both economics and computer science journals. The screening step includes two stages. First, duplicate articles were eliminated, resulting in 135 unique articles that were moved to the next stage, where the relevance of the articles was examined based on their titles and abstracts. The second stage resulted in 84 articles for further consideration. The third step of the Prisma model is eligibility, in which the full text of articles was read by the authors, among which 57 were considered eligible for final review. In fact, at this stage, after reading the abstract and the full text of the articles, the articles that did not develop a model for one of the fields related to economics using the machine learning method were removed. The last step of the Prisma model is the creation of the database and is used for qualitative and quantitative analysis. The database of the current study is comprised of 57 articles, which were all analyzed in this study. Figure 2 illustrates the steps of creating the database of the current study based on the Prisma method. 


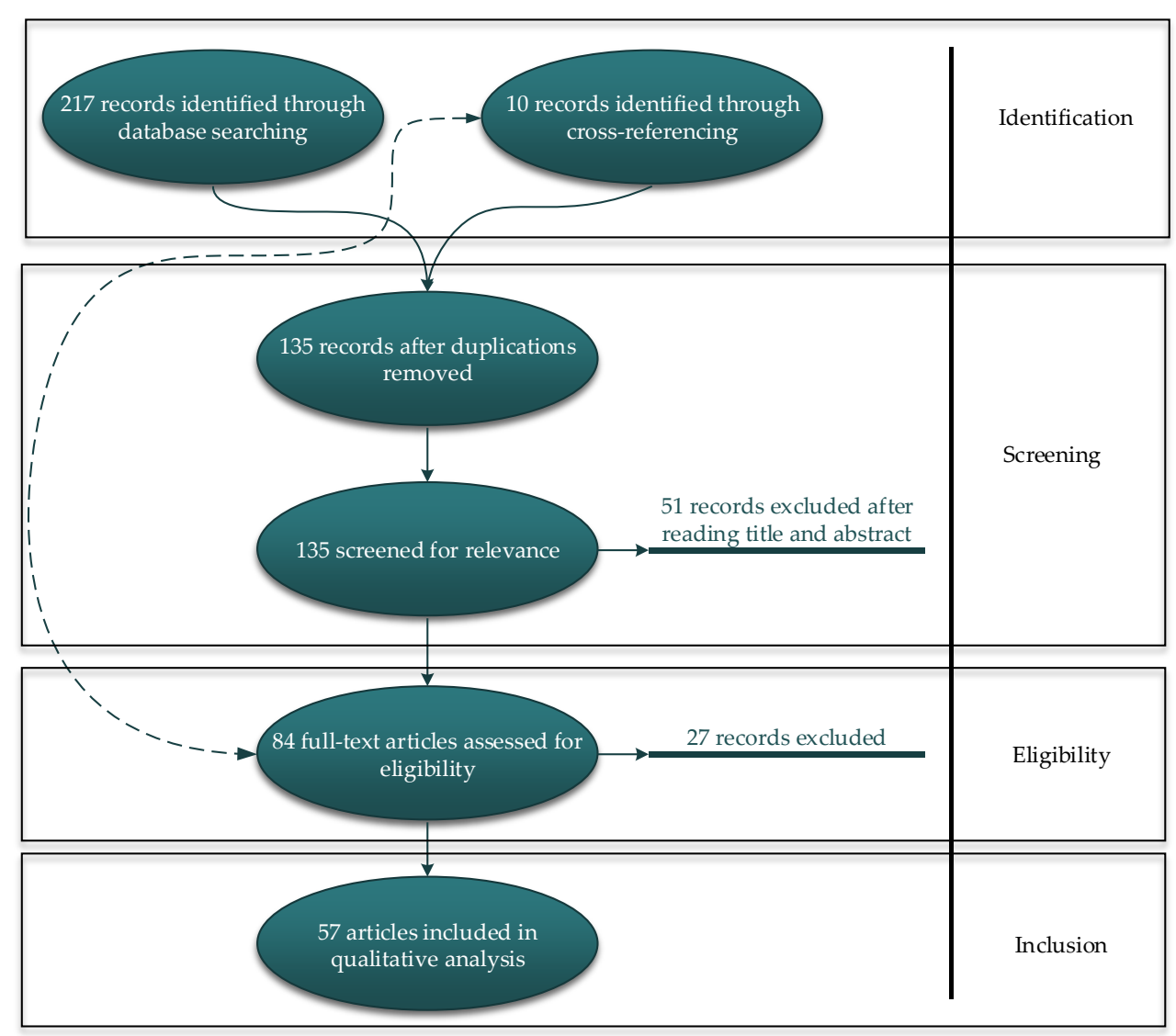

Figure 2. Diagram of systematic selection of the study database using Prisma model

\section{Findings and Discussion}

Figure 2 shows that the database of this study consists of 57 articles that were analyzed and categorized according to two criteria: 1) research/application area, and 2) the method type. Based on the review of articles by application, it was found that these articles were designed to address the issues of five different applications, namely the Stock Market (37 articles), Marketing (6 articles), Ecommerce ( 8 articles), Corporate Bankruptcy ( 3 articles), and Cryptocurrency ( 3 articles). In addition, the articles were analyzed by the type of method, revealing that 42 unique algorithms employed among the 57 reviewed articles (see Figure 3). It was further found that 9 articles used 9 single DL models (Table 8), 18 HDL models (Table 9), 7 hybrid machine learning models (Table 10), and 8 ensemble models (Table 11). In the following section, the identified applications and each of these methods are described in detail. 

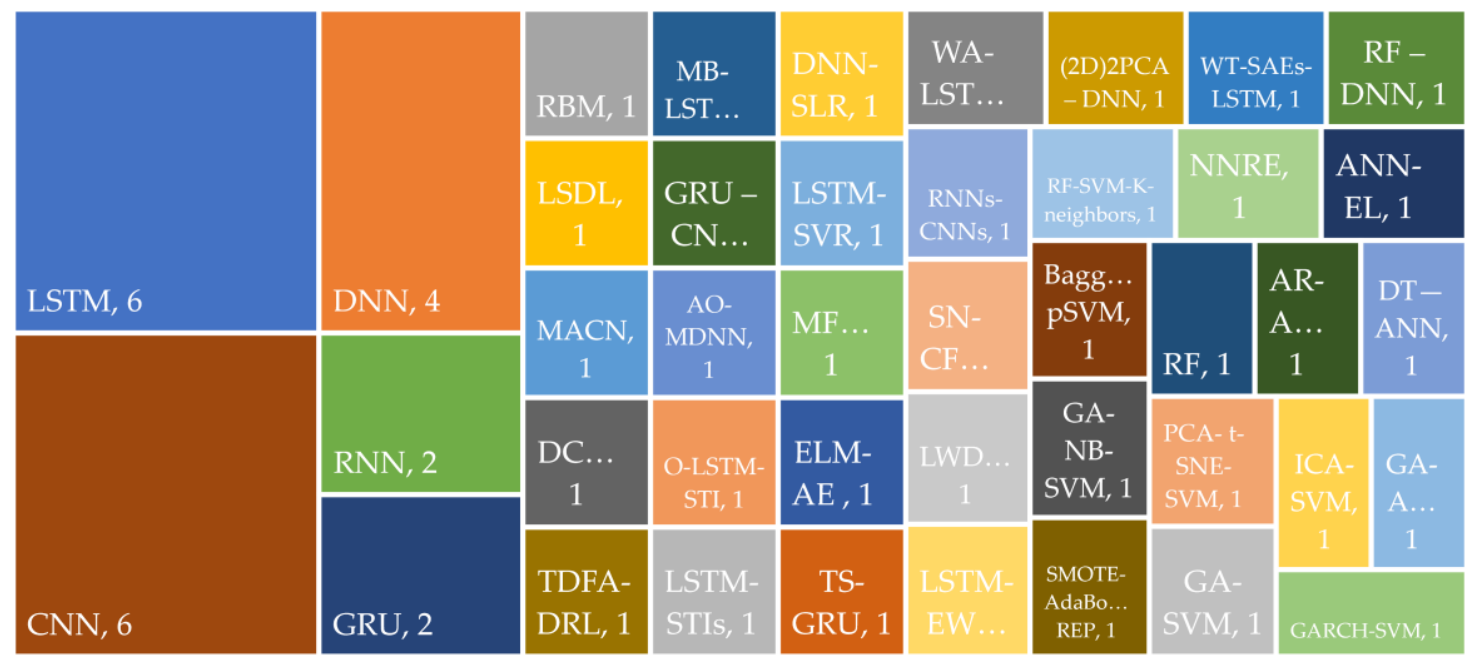

Figure 3. Notable methods of deep learning and hybrid deep learning models applied in economicsrelated fields; the size of the rectangle is proportional to the number of publications (source: WoS)

\subsection{Applications of data science in economics}

\subsubsection{Stock Market}

Applying deep learning in the stock market has become more common than in other areas of economics, considering that most of the research articles reviewed in the present study are classified in this category ( 37 out of 57). Table 2 provides a summary of the articles that employed predictive models in stock market studies, including research objectives, data sources, and applied models of each article. Investment in the stock market is profitable, while the higher the profit, the higher the risk. Therefore, investors always try to determine and estimate the stock value before any action. The stock value is often influenced by a sort of uncontrollable economic and political factors that make it notoriously difficult to identify the future stock market trends. Not only is the nature of the stock market so volatile and complex, the financial time series data are noisy and nonstationary. Thus, the traditional forecasting models are not reliable enough to predict the stock value, and researchers are continuously seeking new methodologies based on DS algorithms to enhance the accuracy of such predictions. Forecasting stock price was found to be the objective of 29 out of 37 articles. Other studies aimed at applying DS in sentiment analysis, or the analysis of the context of texts to extracts subjective information, to identify future trends in the stock market. Besides, portfolio management, algorithmic trading (i.e., using a pre-programmed automated system for trading), automated stock trading, socially responsible investment portfolios, the S\&P 500 index trend prediction, and exchange-tradefund (EFT) options prices prediction were the objectives of other articles that projected to employ DS methods. Financial time series served as the data source of all these studies, except for the studies aimed at sentiment analysis, which used different data sources, such as social media and financial news.

\section{LSTM}

Long short-term memory (LSTM) networks are a special kind of recurrent neural network (RNN) that can overcome the main issue of RNN, i.e., vanishing gradients using the gates to selectively retain relevant information and discard unrelated information. The structure of an LSTM neural network is shown in Figure 4, which is composed of a memory unit $C_{t}$, a hidden state $h_{t}$ and three types of gates, where $t$ indexes the time step. Specifically, for each step $t$, LSTM receives an input $x_{t}$ and the previous hidden state $h_{t-1}$ then calculates the activation of the gates. Finally, the memory unit and the hidden state are updated. The computations involved are described below: 


$$
\begin{gathered}
f_{t}=\sigma\left(W_{f} x_{t}+w_{f} h_{t-1}+b_{f}\right), \\
i_{t}=\sigma\left(W_{i} x_{t}+w_{i} h_{t-1}+b_{i}\right), \\
O_{t}=\sigma\left(W_{o} x_{t}+w_{o} h_{t-1}+b_{o}\right), \\
C_{t}=f_{t} \odot C_{t-1}+i_{t} \odot \sigma_{c}\left(W_{c} x_{t}+w_{c} h_{t-1}+b_{c}\right), \\
h_{t}=O_{t} \odot \tanh \left(C_{t}\right),
\end{gathered}
$$

where $W_{f}, W_{i}$, and $W_{o}$ denote the weights of inputs; and $w_{f}$ and $w_{i}$ indicate weights of recurrent output and biases, respectively. The subscripts $f, i$ and $O$ represent the forget, input, and output gate vectors, respectively; $b_{f}, b_{i}, b_{o}$ denote the biases; and $\odot$ is the element-wise multiplication.

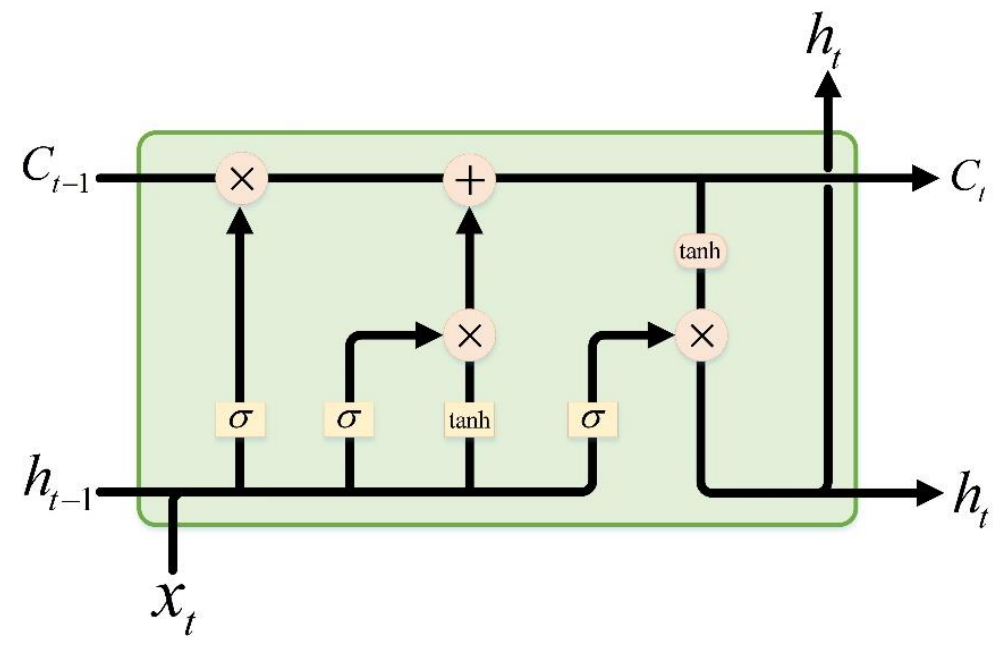

Figure 4. The structure of the LSTM network

Relying on the LSTM algorithm, either a single long short-term memory (LSTM) or a hybrid model of LSTM, many researchers have strived to forecast the stock value. Adapting the LSTM algorithm, Moon and Kim [32] proposed an algorithm to predict the stock market index and volatility. Fischer and Krauss [33] expand the LSTM networks to forecast out-of-sample directional movements in the stock market. The comparison between the performance of their model with the random forest (RF), deep neural network (DNN), and logistic regression classifier (LOG) illustrates the remarkable outperformance of the LSTM model. Tamura et al. [34] introduced a two-dimensional approach to predict the stock values in which the financial technical indices of the Japanese stock market were entered as input data to the LSTM for the prediction, then the data on financial statements of the related companies were retrieved and added to the database. Wang et al. [35] tried to find the best model to predict financial time-series for portfolio management to optimize portfolio formation. They compared the results of LSTM against SVM, RF, DNN, and autoregressive integrated moving average model (ARIMA) and realized that LSTM is more suitable for financial time-series forecasting. Using LSTM, Fister et al. [36] designed a model for automated stock trading. They argue that the performance of LSTM is remarkably higher than the traditional trading strategies, such as passive and rule-based trading strategies. In their case studies, the German blue-chip stock and BMW in the period between 2010 and 2018 formed the data sources.

In addition, there is much evidence in the literature that hybrid LSTM methods outperform the other single DL methods [37]. In the application of the stock market, LSTM has been combined with different methods to develop a hybrid model. For instance, Tamura et al. [34] used LSTM to predict stock price and reported that the results of the accuracy-test outperforms other models in literature. Employing optimal long short-term memory (O-LSTM), Agrawal et al. [38] proposed a model for the stock price prediction using correlation-tensor, which is formed by stock technical indicators (STIs) to optimize the deep learning function. As a result, two predictive models were developed, namely one for price trend prediction and the other for making the buy-sell decision at the end of the day. 
Integrating wavelet transforms (WT), stacked autoencoders (SAEs), and LSTM, Bao, Yue, and Rao [39] established a new method to predict the stock price. In the first stage, WT first eliminates noises to decompose the stock price time series, then predictive features for the stock price are created by SAEs in the next stage. Finally, the LSTM is applied to predict the next day's closing price based on the features generated through the previous stage. Bao et al. [39] claim that their model outperforms the state-of-the-art models in literature in terms of predictive accuracy and profitability performance. To cope with non-linearity and non-stationary characteristics of financial time series, Yan and Ouyang [40] integrate wavelet analysis-LSTM (WA-LSTM) to forecast the daily closing price of the Shanghai Composite Index. Results show that their proposed model outperformed multiple layer perceptron (MLP), SVM, and KNN in finding the patterns in the financial time series. Vo et al. [41] used a multivariate bidirectional-LSTM (MB-LSTM) to develop a deep responsible investment portfolio (DRIP) model for the prediction of stock returns for socially responsible investment portfolios. They applied the deep reinforcement learning (DRL) model to retrain neural networks. Fang, Chen, and Xue [42] developed a methodology to predict the exchange-trade-fund (EFT) options prices. Integrating LSTM model and support vector regression (SVR), They firstly develop two models of LSTM-SVR I and LSTM-SVR II where in LSTM-SVR I the output of LSTM and the final transaction price, buy price, highest price, lowest price, volume, historical volatility, and the implied volatility of the time segment, that considered as factors affecting the price, added as the input of SVR model. Whilst, in LSTM-SVR II, the hidden state vectors of LSTM and the seven factors affecting the option price considered as the SVR's inputs. They also compare the results with the LSTM model and the RF model.

Table 2. Application of data science algorithms in Stock Market

\begin{tabular}{|c|c|c|c|}
\hline Source & Modeling Methods & Data Source & Research Objective \\
\hline Wang et al. [35] & $\begin{array}{l}\text { LSTM Comparing } \\
\text { with SVM, RF, DNN, } \\
\text { and ARIMAs }\end{array}$ & Financial Time Series & Portfolio management \\
\hline Lei et al. [43] & $\begin{array}{l}\text { time-driven feature- } \\
\text { aware and DRL }\end{array}$ & Financial Time Series & Algorithmic trading \\
\hline Vo et al. [41] & $\begin{array}{c}\text { Multivariate } \\
\text { Bidirectional LSTM } \\
\text { Comparing with DRL }\end{array}$ & Financial Time Series & $\begin{array}{l}\text { Socially Responsible } \\
\text { Investment Portfolios }\end{array}$ \\
\hline $\begin{array}{c}\text { Sabeena and Venkata } \\
\text { Subba Reddy [44] }\end{array}$ & GRU - CNN & Financial Time Series & Stock Price Prediction \\
\hline Das and Mishra [45] & $\begin{array}{l}\text { Adam optimizer- } \\
\text { MDNN }\end{array}$ & Financial Time Series & Stock Price Prediction \\
\hline Go and Hong [46] & DNN & Financial Time Series & Stock Price Prediction \\
\hline Agrawal et al. [38] & O-LSTM-STI & Financial Time Series & Stock Price Prediction \\
\hline Gonçalves et al. [47] & $\begin{array}{l}\text { CNN comparing with } \\
\text { DNNC and LSTM }\end{array}$ & Financial Time Series & Stock Price Prediction \\
\hline Moews et al. [48] & DNN-SLR & Financial Time Series & Stock Price Prediction \\
\hline Song et al. [49] & DNN & Financial Time Series & Stock Price Prediction \\
\hline Fang et al. [42] & $\begin{array}{l}\text { LSTM-SVR } \\
\text { comparing with RF } \\
\text { and LSTM }\end{array}$ & Financial Time Series & $\begin{array}{l}\text { Exchange-trade-fund } \\
\text { (EFT) Options Prices } \\
\text { Prediction }\end{array}$ \\
\hline Long et al. [50] & $\begin{array}{c}\text { MFNN (CNN and } \\
\text { RNN) }\end{array}$ & Financial Time Series & Stock Price Prediction \\
\hline Fister et al. [36] & LSTM & Financial Time Series & $\begin{array}{c}\text { Automated Stock } \\
\text { Trading }\end{array}$ \\
\hline Rajesh [51] & RF, SVM and KNN & Financial Time Series & Stock Price Prediction \\
\hline Moon and Kim [32] & LSTM & Financial Time Series & Stock Price Prediction \\
\hline
\end{tabular}


Sim Kim, and Ahn [52]

Agrawal et al. [53]

Tashiro et al. [54]

Sirignano and Cont

[55]

Weng et al. [56]

Preeti et al. [57]

Sohangir et al. [58]

Fischer and Krauss

Lien Minh et al. [59]

Das et al. [60]

Yan and Ouyang [40]

Kim et al. [61]

Faghihi-Nezhad and

Minaei-Bidgoli [62]

Tamura et al. [34]

Chong et al. [63]

Dingli and Fournier [64]

Singh and Srivastava

Bao et al. [39]

Shekhar and Varshney

[66]

Ahmadi et al. [67]

Ebadati and Mortazavi [68]

Johari et al. [69]
CNN comparing with ANN and SVM

LSTM-STIs

$\mathrm{CNN}$

LSDL

BRT comparing with

NNRE, SVRE, and RFR

ELM-AE comparing with GARCH, GRNN, MLP, RF and GRDH

CNN comparing with doc2vec and LSTM

LSTM comparing with RF, DNN and LOG

two-stream GRU

DNN

wavelet analysis with

LSTM, comparing

with SVM, KNN and

MLP

MACN

EL-ANN

LSTM

DNN comparing

with PCA,

Autoencoder, and RBM

CNN

(2D)2PCA - DNN comparing with RBFNN WT-SAEs-LSTM

GA-SVM

ICA- SVM

GA-ANN

GARCH-SVM
Financial Time Series

Financial Time Series

Financial Time Series

Financial Time Series

Financial Time Series

Stock Price Prediction

Financial Time Series

Stock Price Prediction

Social media

Financial Time Series

Financial news

Financial Time Series

Financial Time Series

Financial Time Series

Financial Time Series

Financial Time Series

Financial Time Series

Stock Price Prediction

Financial Time Series

Stock Price Prediction

Financial Time Series

Stock Price Prediction

Financial Time Series

Stock Price Prediction

Financial Time Series

Stock Price Prediction

Financial Time Series

Stock Price Prediction

Financial Time Series

Stock Price Prediction

Financial Time Series

Stock Price Prediction

DNN

Deep neural network (DNN), which is composed of multiple levels of nonlinear operations, and each layer only receives the connections from its previous training layer as shown in Figure 5, adapted from [49]. Suppose $X$ be the input data, and $w_{j}, j=1,2, \ldots, k$ be a filter bank, where $k$ is the number of layers. The multi-layer features of the DNN can be computed as:

$$
f(X)=f_{k}\left(\cdots f_{2}\left(f_{1}\left(X ; w_{1}\right) ; w_{2}\right) \cdots, w_{k}\right) .
$$


Currently, DNN has widely applied in the stock market to identify the trends and patterns among the financial time series data. Go and Hong [46] applied the DNN method to predict the stock value. They firstly train the method by the time series data and then test and confirm the predictability of their model. Song et al. [49] develop DNN using 715 novel input-features to forecast the stock price fluctuation. They also compare the performance of their model with the other models that include simple price-based input-features. For predicting the stock market behavior, Chong, Han, and Park [63] examine the performance of DNN. They consider high-frequency intraday stock returns as the input in their model. They analyzed the predictability of principal component analysis (PCA), autoencoder, and RBM. According to their results, DNN has good predictability with the information they receive from the residuals of the autoregressive mode. Whilst, applying the autoregressive model to the residuals of the network cannot contribute to the predictability of the model. Besides, Chong et al. [63] found out applying covariance-based market structure analysis to the predictive network remarkably increase the covariance estimation. Das et al. [60] use DNN to predict the future trends of the S\&P 500 Index. Their results show that their model can poorly forecast the behavior of the underlying stocks in the S\&P 500 index. They believe that randomness and nonstationarity are the reasons that make hard the predictability of this index.

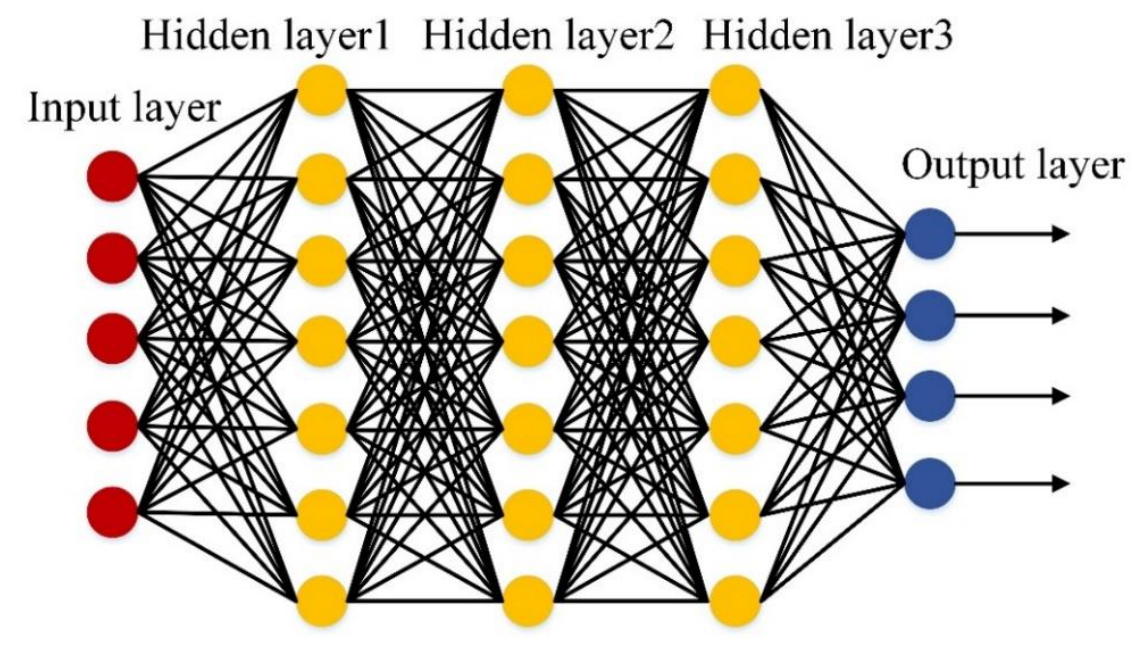

Figure 5. Structure of the deep neural network

Besides, hybrid methods that are constructed based on DNN have been reported to be very accurate in the financial time series data. For example, Das and Mishra [45] proposed an advanced model to plan, analyze, and predict the stock value, using a multilayer deep neural network (MDNN) optimized by Adam optimizer (AO) to find the patterns among the stock values. Moews et al. [48] proposed a method to predict the behavior of the stock market, as a complex system with a massive number of noisy time series. Their model integrates DNN and stepwise linear regressions (SLR). Moews et al. [48] considered regression slopes as trend strength indicators for a given time interval. To predict the Google stock price, Singh and Srivastava [65] compared two integrated models, i.e., 2directional 2-dimensional principal component analysis-DNN ((2D)2PCA-DNN) and (2D)2PCAradial basis function neural network (RBFNN). According to their results, the (2D) 2PCA-DNN model has higher accuracy in predicting the stock price. They also compared their results with the RNN model and reported that the predictability of (2D)2PCA-DNN outperforms RNN as well. 


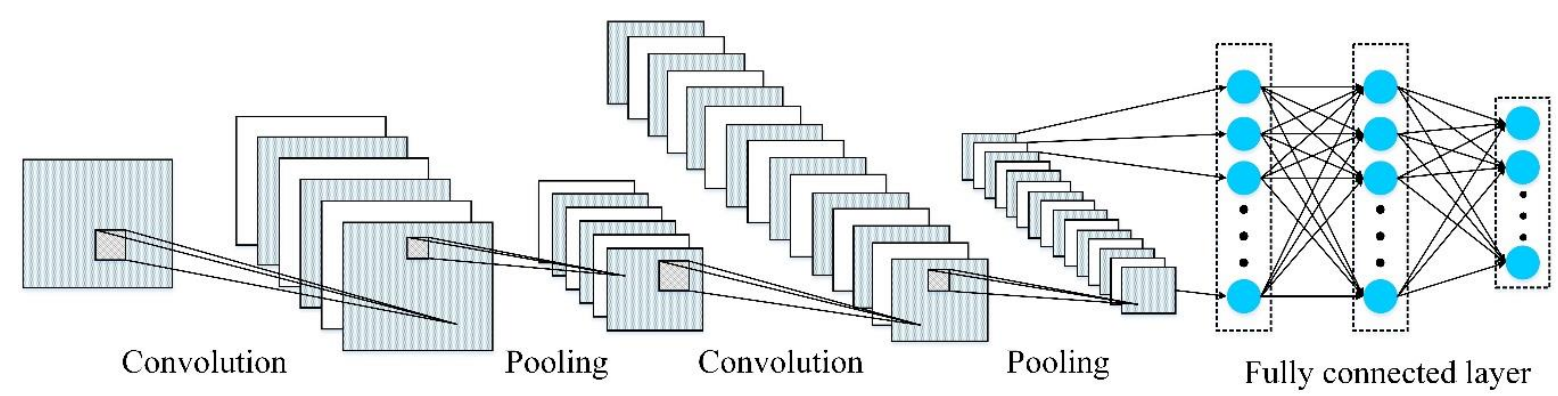

Figure 6. Structure of the CNN

Convolutional neural network $(\mathrm{CNN})$ is one of the most popular methods in deep learning and is widely applied in various fields [70-73], such as classification, language processing, and object detection. A classical structure of $\mathrm{CNN}$ is presented in Figure 6, adapted from [71], which mainly consists of three components, i.e., convolution layers, pooling layers, and fully connected layers. Different layers have different roles in the training process and are discussed in more detail below:

Convolutional layer: This layer is composed of a set of trainable filters, which are used to perform feature extraction. Suppose $X$ is the input data and there are $k$ filters in convolutional layers, then the output of the convolutional layer can be determined as follows:

$$
y_{j}=\sum_{i} f\left(x_{i} * w_{j}+b_{j}\right), j=1,2, \ldots, k
$$

where $w_{j}$ and $b_{j}$ are the weight and bias, respectively; and $f(\cdot)$ denotes an activation function. * represents the convolution operation.

Pooling layer: In general, this layer is used to decrease the dimensions of the obtained feature data and network parameters. Currently, max pooling and average pooling are the most widely used methods. Let $S$ be a $p \times p$ window size, then the average pooling operation can be expressed as follows:

$$
z=\frac{1}{N} \sum_{(i, j) \in S} x_{i j}, i, j=1,2 \ldots, p
$$

where $x_{i j}$ indicates the activation value at $(i, j)$; and $N$ is the total number of elements in $S$.

Fully connected layer: Following the last pooling layer, the fully connected layer is utilized to reshape the feature maps into a 1-D feature vector, which can be expressed as:

$$
Y=\sum_{i} f(w z+b)
$$

where $Y$ and $\mathrm{z}$ denote the output vector and input features; and $w$ and $b$ represent the weight and bias of the fully connected layer, respectively.

Recently, CNN has been extensively applied by many researchers for predicting stock values using financial time series data. Sim et al. [52] developed a model to predict the stock price by adapting CNN. Their results reveal that the predictive performance of CNN demonstrated a better performance in forecasting stock price than ANN and SVM. Tashiro et al. [54] first criticized the current models for the price prediction in the stock markets in that the properties of market orders are ignored. Therefore, they constructed a $\mathrm{CNN}$ architecture integrating order-based features to predict the mid-price trends in the stock markets. Their results prove that adding the features of orders to the model increased its accuracy. Dingli and Fournier [64] applied CNN to predict the future movement of stock prices and found that the predictive accuracy of their model was $65 \%$ in predicting the following month's price and was $60 \%$ for the following week's price. Gonçalves et al. [47] compared the results of the prediction of CNN, LSTM, and deep neural network classifier (DNNC) for finding the best model to predict the price trends in the exchange markets. Their findings reveal that $\mathrm{CNN}$, on average, has the best predictive power. Sohangir et al. [58] compared the performance 
of several neural network models, including CNN, LSTM, and doc2vec, for sentiment analysis among the posts and opinions of the experts in StockTwits to predict the movements in the stock markets. Their results disclose that $\mathrm{CNN}$ has the highest accuracy in predicting the sentiment of experts.

To increase the accuracy of $\mathrm{CNN}$, some researchers integrated $\mathrm{CNN}$ with other models and proposed new hybrid models. For example, by integrating gated recurrent unit (GRU) and CNN, Sabeena et al. [44] introduced a hybrid DL model to predict financial fluctuations in the real-time stock market that is able to process the real-time data from online financial sites. GRUs are developed based on the RNN architectures. They represent a simpler implementation for LSTMs to address gradient functions and learn long-range dependencies. To predict the price movement from financial time series samples, Long et al. [50] introduced an end-to-end model called multi-filters neural network (MFNN) that incorporates $\mathrm{CNN}$ and recurrent neural network (RNN).

\section{Other algorithms}

In addition to LSTM, DNN, and CNN, other DS methods have been employed for predicting stock value using time series data. For example, Sirignano and Cont [55] developed an LSDL model to study the USA market quotes and transactions. Their results disclose that there is a universal and stationary relationship between order flow history and price trends. Kim et al. [61] proposed a multiagent collaborated network (MACN) model to optimize financial time series data, claiming that their model can share and generalize the experience of agents in stock trading.

Besides, various other hybrid methods have been applied by the researchers for financial time series data. For instance, to predict the stock price trends, Lien Minh et al. [59] developed and trained a two-stream GRU (TS-GRU) network and Stock2Vec model to analyze the sentiments in the financial news and their relationship with the financial prices, based on their belief that financial news and sentiment dictionaries affect the stock prices. Their findings support the outperformance of their model in comparison with the current models. Lien Minh et al. [59] also claim that Stock2Vec is highly efficient in financial datasets. Lei et al. [43] combined deep learning models and reinforcement learning models to develop a time-driven feature-aware (TDFA) jointly deep reinforcement learning model (TFJ-DRL) for financial time-series forecasting in algorithmic trading. Preeti et al. [57] introduced an extreme learning machine (ELM)-auto-encoder (AE) model to identify patterns in the financial time series. They tested the accuracy of their model in the time series data of gold price and crude oil price and compared their results with those of generalized autoregressive conditional heteroskedasticity (GARCH), GRNN, MLP, RF and group method of data handling (GRDH). The result of the mean square error (MSE) test proved that the performance of their model was higher than the existing methods.

In addition to the hybrid deep learning models, four articles applied hybrid machine learning models to financial time series data. Shekhar and Varshney [66] integrated a hybrid model of genetic algorithm-SVM (GV-SVM) with sentiment analysis to predict the future of the stock market. Using quantitative empirical analysis, they proved that the combination of sentiment analysis with GVSVM increased the model's accuracy by $18.6 \%$ and reported the final model's accuracy of about $89.93 \%$. Ahmadi et al. [67] compared the performance of two hybrid machine learning models in predicting the timing of the stock markets, using imperialist competition algorithm-SVM (ICASVM), and SVM-GA. Their results exposed that SVM-ICA has a higher performance compared with SVM-GA in the prediction of stock market trends for periods of 1-6 days. To predict stock prices using financial time series data, Ebadati and Mortazavi [68] applied a hybrid model by integrating GA-ANN, where GA was employed to select ANN features and optimize parameters. Their study suggests that this hybrid machine learning model has an improved sum square error (SSE) (i.e., performance accuracy) by $99.99 \%$ and improved time (i.e., speed accuracy) by $90.66 \%$. Johari et al. [69] compared the accuracy and performance of GARCH-SVM and GARCH-ANN models in the financial time series data for stock price forecasting. They found that GARCH-SVM outperformed GARCH-ANN, SVM, ANN, and GARCH based on MSE and RMSE accuracy metrics.

Rajesh et al. [51] used ensemble learning to predict the future stock trends by applying heat maps and ensemble learning to financial data of the top 500 companies in the S\&P stock exchange. Their 
results show that the combination of RF, SVM, and K-neighbors classifiers had the most accurate results, and the accuracy of the proposed model was $23 \%$ higher than a single classifier labeling prediction model. Weng et al. [56] aimed to design a financial expert system to forecast short-term stock prices. To analyze the collected data and predict stock prices, they employed four machine learning ensemble methods, namely neural network regression ensemble (NNRE), support vector regression ensemble (SVRE), boosted regression tree, and random forest regression (RFR). Using Citi Group stock (\$C) data, they were able to forecast the 1-day ahead price of 19 stocks from different industries. Weng et al. [56] claim that the boosted regression tree (BRT) outperformed other ensemble models with a considerably better mean absolute percent error (MAPE) than those reported in literature. Faghihi-Nezhad and Minaei-Bidgoli [62] employed ensemble learning and ANN to develop a two-stage model to predict the stock price. They first predicted the direction of the next price movement and then created a new training dataset to forecast the stock price. They used a genetic algorithm (GA) optimization and particle swarm optimization (PSO) to optimize the results of each stage. The results ultimately reveal that the accuracy of their model in prediction of stock price outperformed other models in the literature.

Reviewing the articles categorized in the stock market category reveals that, although the research objectives of the articles are different, most utilized financial time series data (35 out of 37 articles). Only 2 articles used the financial news and social media as the data source to determine the future trends in the stock market (see Table 3).

Table 3. Classification of Articles Using Data Science by Research Purpose and Data Source in the Stock Market Section

\begin{tabular}{|c|c|c|}
\hline Research Objective & Data Source & $\begin{array}{l}\text { Number of } \\
\text { documents }\end{array}$ \\
\hline Stock Price Prediction & Financial Time Series & 29 \\
\hline Sentiment Analysis & $\begin{array}{l}\text { Financial News, } \\
\text { Social Media }\end{array}$ & 2 \\
\hline Portfolio management & Financial Time Series & 1 \\
\hline Algorithmic trading & Financial Time Series & 1 \\
\hline $\begin{array}{l}\text { Socially Responsible } \\
\text { Investment Portfolios }\end{array}$ & Financial Time Series & 1 \\
\hline Automated Stock Trading & Financial Time Series & 1 \\
\hline $\begin{array}{c}\text { The S\&P } 500 \text { Index Trend } \\
\text { Prediction }\end{array}$ & Financial Time Series & 1 \\
\hline $\begin{array}{l}\text { Exchange-trade-fund } \\
\text { (EFT) Options Prices } \\
\text { Prediction }\end{array}$ & Financial Time Series & 1 \\
\hline
\end{tabular}

\subsubsection{Marketing}

Studying the purpose of the articles discloses that DS algorithms were mostly used for the purpose of studying customer behavior and promotional activities, which is why these articles are 
classified in group labeled marketing. As seen in Table 4, two studies applied a single DL method, and three used hybrid DL methods. In addition, these studies used various data sources, such as customer time series data, case studies, and social media. For example, Paolanti et al. [74] employed deep convolutional neural network (DCNN) to develop a mobile robot, so-called ROCKy, to analyze real-time store heat maps of retail store shelves for detection of the shelf-out-of-stock (SOOS) and promotional activities during working hours. Dingli, Marmara, and Fournier [75] investigated solutions to identify the patterns and features among transactional data to predict customer churn within the retail industry. To do so, they compared the performance of $\mathrm{CNN}$ and restricted Boltzmann machine (RBM), realizing the RBM outperformed in customer churn prediction.

Table 4. Application of data science algorithms in Marketing

\begin{tabular}{|c|c|c|c|}
\hline Source & $\begin{array}{l}\text { Modeling } \\
\text { Methods }\end{array}$ & Data Source & Research Objective \\
\hline Ładyżyński et al. [76] & RF - DNN & $\begin{array}{c}\text { Time Series data } \\
\text { of Customers }\end{array}$ & Customer Behavior \\
\hline Ullah et al. [77] & $\mathrm{RF}$ & $\begin{array}{l}\text { Time Series data } \\
\text { of Customers }\end{array}$ & Customer Behavior \\
\hline Paolanti et al. [74] & DCNN & Primary Data & $\begin{array}{l}\text { Detection of Shelf Out } \\
\text { of Stock (SOOS) and } \\
\text { Promotional } \\
\text { Activities }\end{array}$ \\
\hline Agarwal [78] & RNNs- CNNs & Social media & Sentiment Analysis \\
\hline $\begin{array}{l}\text { Shamshirband et al. } \\
\text { [79] }\end{array}$ & SN-CFM & Social media & Customer behavior \\
\hline Dingli et al. [75] & RBM & Primary Data & Customer behavior \\
\hline
\end{tabular}

On the other hand, RF-DNN, RNN-CNN, and similarity, neighborhood-based collaborative filtering model (SN-CFM) are hybrid models that researchers used to study customer behavior. Ładyżyński et al. [76], for instance, employed Random Forest (RF) and DNN methods and customers' historical transactional data to propose a hybrid model that can predict the customers' willingness to purchase credit products from the banks. Ullah et al. [77] used the RF algorithm to predict churn customers and to formulate strategies for customer relationship management to prevent churners. Ullah et al. [77] explained that a combination of churn classification, utilizing the RF algorithm and customer profiling using k-means clustering, increased their model's performance. Agarwal [78] integrated RNN and CNN to develop a model for sentiment analysis. According to Agarwal [78], sentiment analysis is the best approach to receive the customers' feedback. He tested his proposed model using social media data and believes that the result of the sentiment analysis provides guidance to the business to improve the quality of their service and presents evidence for the startups to improve customer experience. Shamshirband et al. [79] proposed SN-CFM to predict consumer preferences according to the similarity of features of users and products that are acquired from the Internet of Things and social networks.

\subsubsection{E-commerce}

Another category that emerged after reviewing the articles is labeled e-commerce, where the authors employed data science models to address problems in the e-commerce sector. A summary of these studies is presented in Table 5. Based on the GRU model, Lei [80] designed a neural network investment quality evaluation model to support the decision-making related to investment in ecommerce. Their proposed model is able to evaluate different index data that provides a better picture to investors. Leung et al. [81] argue that the ability to handle orders and logistics management is one of the major challenges in e-commerce. Therefore, using a hybrid autoregressive-adaptive neuro-fuzzy inference system (AR-ANFIS), they developed a prediction model for B2C e-commerce order arrival. According to their results, their proposed hybrid model can successfully forecast e- 
order arrivals. Cai et al. [82] used deep reinforcement learning to develop an algorithm to address allocating impression problems on e-commerce websites, such as www.taobao.com, www.ebay.com, and www.amazon.com. In this algorithm, the buyers are allocated to the sellers based on the buyer's impressions and the seller's strategies in a way that maximizes the income of the platform. To do so, they applied GRU, and their findings show that GRU outperforms the deep deterministic policy gradient (DDPG). Ha, Pyo, and Kim [83] applied RNN to develop a deep categorization network (Deep $\mathrm{CN}$ ) for item categorization in e-commerce, which refers to classifying the leaf category of items from their metadata. They used RNN to generate features from text metadata and categorize the items accordingly. $\mathrm{Xu}$ et al. [84] designed an advanced credit risk evaluation system for ecommerce platforms to minimize the transaction risk associated with buyers and sellers. To this end, they employed a hybrid machine learning model combined with decision tree-ANN (DT-ANN) and found that this model has high accuracy and outperforms other hybrid machine learning models, such as DT-Logistic Regression and DR-dynamic Bayesian network.

Selling products online has unique challenges, for which data science has been able to provide solutions. To increase the buyer's trust in the quality of the products and to buy online, Saravanan and Charanya [85] designed an algorithm where the products are categorized according to several criteria, including reviews and ratings of other users. By integrating a hybrid feature extraction method principle component analysis (PCA) and $t$-distributed stochastic neighbor embedding ( $t$ SNE) with SVM using lexicon-based method, Saravanan and Charanya [85] also proposed a model that separates the products from the large collection of different products based on characteristics, best product ratings, and positive reviews. Wang, Mo, and Tseng [86] used RNN to develop a personalized product recommendation system on e-commerce websites. The result of their model disclose the outperformance of RNN to K-nearest neighbors (KNN). Wu and Yan [87] claim that the main assumption of the current production recommender models for e-commerce websites is that all historical data of users are recorded, while in practice, many platforms fail to record such dada. Therefore, they devised a list-wise DNN (LWDNN) to model the temporal online behaviors of users and provide recommendations for anonymous users.

Table 5. Application of data science algorithms in e-commerce

\begin{tabular}{|c|c|c|c|}
\hline Source & $\begin{array}{l}\text { Model } \\
\text { ing } \\
\text { Metho } \\
\text { ds }\end{array}$ & $\begin{array}{c}\text { Data } \\
\text { Source }\end{array}$ & Research Objective \\
\hline Lei [80] & GRU & $\begin{array}{c}\text { Financial } \\
\text { Time } \\
\text { Series }\end{array}$ & $\begin{array}{l}\text { Investment Quality } \\
\text { Evaluation Model }\end{array}$ \\
\hline Leung et al. [81] & $\begin{array}{c}\text { AR- } \\
\text { ANFIS }\end{array}$ & $\begin{array}{c}\text { Primary } \\
\text { Data }\end{array}$ & $\begin{array}{l}\text { Order Arrival } \\
\text { Prediction }\end{array}$ \\
\hline Cai et al. [82] & GRU & $\begin{array}{c}\text { Customers } \\
\text { Time } \\
\text { Series }\end{array}$ & $\begin{array}{c}\text { Impression } \\
\text { Allocation Problem }\end{array}$ \\
\hline Ha et al. [83] & RNN & $\begin{array}{c}\text { Primary } \\
\text { Data }\end{array}$ & Item Categorization \\
\hline Xu et al. [84] & $\begin{array}{l}\mathrm{DT}- \\
\mathrm{ANN}\end{array}$ & $\begin{array}{l}\text { Credit } \\
\text { Data }\end{array}$ & $\begin{array}{c}\text { Dynamic Credit Risk } \\
\text { Evaluation }\end{array}$ \\
\hline
\end{tabular}




$\begin{array}{lccc}\begin{array}{c}\text { Saravanan and } \\ \text { Charanya [85] }\end{array} & \begin{array}{c}\text { PCA- } \\ \text { t-SNE- } \\ \text { SVM }\end{array} & \begin{array}{c}\text { Primary } \\ \text { Data }\end{array} & \begin{array}{c}\text { Product } \\ \text { Recommendation }\end{array} \\ \text { Wang et al. [86] } & \text { RNN } & \text { Primary } & \text { Product } \\ & & \text { Data } & \text { Recommendation } \\ \text { Wu and Yan [87] } & \text { CWD } & \text { Customers } & \\ & \text { NN } & \text { Time } & \text { Product } \\ & & \text { Series } & \text { Recommendation }\end{array}$

\subsubsection{Cryptocurrency}

The decision-making process related to investing in the cryptocurrencies is similar to investing in the stock market, where the prediction of future value is very determinant and effective on the investment decisions. Applying machine learning and DL models to predict the trends of cryptocurrency prices is an attractive research problem that is emerging in the literature (see Table 6). For example, Lahmiri and Bekiros [88] applied deep learning methods for the prediction of the price of cryptocurrencies, including Bitcoin, Digital Cash, and Ripple, and compared the predictive performance of LSTM and GRNN. Their findings disclose that the LSTM model has a better performance in comparison with GRNN. Altan, Karasu, and Bekiros [89] claim that integrating LSTM and empirical wavelet transform (EWT) improves the performance of LSTM in forecasting the digital currency price by testing their proposed model using Bitcoin, Ripple, Digital Cash, and Litecoin time series data. Jiang and Liang [90] developed a CNN model to predict the price of Bitcoin as a cryptocurrency example. They train their proposed model using historical data of financial assets prices and used portfolio weights of the set as the output of their model.

Table 6. Application of data science algorithms in Cryptocurrency

\begin{tabular}{cccc}
\hline Source & Modeling Methods & Data Source & Research Objective \\
\hline $\begin{array}{c}\text { Lahmiri and } \\
\text { Bekiros [88] }\end{array}$ & LSTM comparing with & & Cryptocurrencies \\
& GRNN & Financial Time Series & $\begin{array}{c}\text { Price prediction } \\
\text { Cryptocurrencies }\end{array}$ \\
Altana et al. [89] & LSTM-EWT & Financial Time Series & Price prediction \\
Jiang and Liang & & & Cryptocurrencies \\
[90] & CNN & Financial Time Series & Price prediction \\
\hline
\end{tabular}

\subsubsection{Corporate bankruptcy prediction}

Corporate bankruptcy prediction has become an important tool to evaluate the future financial situation of a company. Utilizing machine learning-based methods is widely recommended to address bankruptcy prediction problems. Such as done by Chen, Chen, and Shi [91], who utilized bagging and boosting ensemble strategies and develop two models of Bagged-proportion support vector machines (pSVM) and Boosted-pSVM. Using datasets of UCI and LibSVM, they test their models and explain that ensemble learning strategies increased the performance of the models in bankruptcy prediction. Lin, Lu, and Tsai [92] believe that finding the best match of feature selection and classification techniques improves the prediction performance of bankruptcy prediction models. Their results reveal that the genetic algorithm as the wrapper-based feature selection method and the combination of the genetic algorithm with the naïve Bayes and support vector machine classifiers had a remarkable predictive performance. Lahmiri et al. [93], to develop an accurate model for forecasting corporate bankruptcy, compare the performance of different ensemble systems of AdaBoost, LogitBoost, RUSBoost, subspace, and bagging. Their finding reveals that the AdaBoost 
model has been effective in terms of short-time data processing and low classification error, and limited complexity. Faris et al. [94] investigate the combination of re-sampling (oversampling) techniques and multiple features of election methods to improve the accuracy of bankruptcy prediction methods. According to their results, employing SMOTE oversampling technique and AdaBoost ensemble method using reduced error pruning (REP) tree provides reliable promising results to bankruptcy prediction. A summary of these research articles is presented in Table 7.

Table 7. Application of data science algorithms in corporate bankruptcy prediction

\begin{tabular}{cccc}
\hline Source & Modeling Methods & Data Source & Research Objective \\
\hline $\begin{array}{c}\text { Chen et al. } \\
\text { [91] }\end{array}$ & $\begin{array}{c}\text { Bagged-pSVM and Boosted- } \\
\text { pSVM }\end{array}$ & $\begin{array}{c}\text { UCI and LibSVM } \\
\text { datasets }\end{array}$ & $\begin{array}{c}\text { bankruptcy } \\
\text { prediction }\end{array}$ \\
Lin et al. [92] & $\begin{array}{c}\text { Genetic Algorithm with the } \\
\text { Naïve Bayes and SVM } \\
\text { classifiers }\end{array}$ & $\begin{array}{c}\text { Australian credit, } \\
\text { German credit, and } \\
\text { Taiwan bankruptcy } \\
\text { datasets }\end{array}$ & $\begin{array}{c}\text { bankruptcy } \\
\text { prediction }\end{array}$ \\
Lahmiri et & AdaBoost & University of \\
al. [93] & & $\begin{array}{c}\text { California Irvine (UCI) } \\
\text { Machine Learning }\end{array}$ & $\begin{array}{c}\text { bankruptcy } \\
\text { prediction }\end{array}$ \\
Faris et al. & RMOTE- AdaBoost- REP Tree & Infotel database & bankruptcy \\
[94] & SMrediction
\end{tabular}

\subsection{Applied data science methods in the Economics}

\subsubsection{Deep learning methods}

The new generation of deep learning methods rooted in the work of Hinton et al. [95]. Hinton et al. [95] develop, for the first time, a two-step approach in which the deep learning algorithms are firstly trained and then fine-tune the model in a back-through process. The advantage of DL models compared to other ML models is that DL models can effectively identify high-level features and representations (the outputs) from a large diverse data sample (inputs). Employing an unsupervised pre-training and a supervised fine-tuning approach, the deep learning models extract hierarchical features from the inputs to classify the patterns of the data [96]. The ability of DL algorithms in prediction and finding the patterns among the raw data has grabbed the attraction of many researchers from various fields. As explored above the stock price prediction, e.g. [32-34] and consumer behavior, e.g.[75] have well benefited with a wide range of a deep learning algorithms. Among the methods, the LSTM, CNN, and DNN are respectively the most popular DL [32-34]. LSTM method has also been very popular for predicting financial time series. The DL applications to portfolio management [35], automated stock trading [36], and cryptocurrencies price prediction [88] have also been relatively popular.

Similar to LSTM, the CNN algorithm is applied mainly for financial time series data to stock price prediction $[47,52,54,64]$ and cryptocurrencies price prediction [90]. CNN algorithm is also used for analyzing social media data for sentiment analysis [58]. The DNN algorithm, likewise the LSTM, is only used to analyze financial time series data to predict stock prices $[46,49,63]$ and the S\&P 500 Index trend prediction [60]. The GRU algorithm, which is another DL model, is applied in the ecommerce section to analyze financial time series [80] and customer time series [82]. RNN is a DL algorithm applied to analyze primary data for item categorization [83] and product recommendation [86]. Large-scale deep learning (LSDL) and MACN are DL algorithms that are used to analyze financial time series data to predict stock price [55,61]. Ultimately, it is found that DCNN and RBM applied for analyzing primary data for respectively promotional activities [74] and customer behavior forecasting [75] (see Table 8). 
Table 8. List of single deep learning methods employed in Economics related fields

\begin{tabular}{|c|c|c|c|c|}
\hline \multirow[b]{2}{*}{ Method } & \multicolumn{4}{|c|}{ Applications } \\
\hline & Stock Market & Marketing & Cryptocurrency & $\begin{array}{c}\text { E- } \\
\text { Commerce }\end{array}$ \\
\hline LSTM & $\begin{array}{c}\text { Moon and Kim [32], Fischer and Krauss } \\
\text { [33], Tamura et al. [34], Wang et al. [35], } \\
\text { Fister et al. [36], }\end{array}$ & - & $\begin{array}{l}\text { Lahmiri and } \\
\text { Bekiros [88] }\end{array}$ & - \\
\hline $\mathrm{CNN}$ & $\begin{array}{l}\text { Gonçalves et al. [47], Sim Kim, and Ahn } \\
\text { [52], Tashiro et al. [54], Sohangir et al. } \\
\text { [58], Dingli and Fournier [64], }\end{array}$ & - & $\begin{array}{c}\text { Jiang and Liang } \\
\text { [90] }\end{array}$ & - \\
\hline DNN & $\begin{array}{c}\text { Go and Hong [46], Song et al. [49], Das et } \\
\text { al. [60], Chong et al. [63] }\end{array}$ & - & - & - \\
\hline GRU & - & - & - & $\begin{array}{l}\text { Lei [80], Cai } \\
\text { et al. [82] }\end{array}$ \\
\hline RNN & - & - & - & $\begin{array}{l}\text { Ha et al. } \\
\text { [83], Wang } \\
\text { et al. [86] }\end{array}$ \\
\hline LSDL & Sirignano and Cont [55] & - & - & - \\
\hline MACN & Kim et al. [61] & - & - & - \\
\hline DCNN & - & $\begin{array}{l}\text { Paolanti et } \\
\text { al. [74] }\end{array}$ & - & - \\
\hline RBM & - & $\begin{array}{l}\text { Dingli et al. } \\
\quad[75]\end{array}$ & - & - \\
\hline
\end{tabular}

\subsubsection{Hybrid deep learning methods}

Hybrid deep neural networks are architectures that apply generative and discriminative components at the same time. Hybrid models combine machine learning models or combine a machine learning model with an optimization model to improve the predictivity of the deep learning model [9]. The findings of the literature review in the study reveal that hybrid deep learning (HDL) models are widely applied in the field of economics. On the other hand, Figure 8 illustrates that the accuracy of such models is reported higher than the single DL models. The various HDL models used among the papers reviewed in this study are summarized in Table 9.

Table 9. List of hybrid deep learning models employed in Economic related fields

\begin{tabular}{ccc}
\hline Tpplication & Source \\
\hline TDFA-DRL & Lei et al. [43] \\
MB-LSTM & Vo et al. [41] \\
GRU - CNN & Sabeena and Venkata Subba Reddy [44] \\
AO-MDNN & Das and Mishra [45] \\
OLSTM-STI & Agrawal et al. [38] \\
DNN-SLR & Moews et al. [48] \\
LSTM-SVR & Fang et al. [42] \\
MFNN (CNN and RNN) & Long et al. [50] \\
LSTM-STIs & Agrawal et al. [53] \\
ELM-AE & Preeti et al. [57] \\
TS-GRU & Lien Minh et al. [59] \\
WA-LSTM & Yan and Ouyang [40] \\
(2D)2PCA - DNN & Singh and Srivastava [65] \\
WT-SAEs-LSTM & Bao et al. [39] \\
RNNs- CNNs & Agarwal [78] \\
SN-CFM & Shamshirband, et al. [79]
\end{tabular}




$\begin{array}{ccc}\text { E-commerce } & \text { LWDNN } & \text { Wu and Yan [87] } \\ \text { Cryptocurrency } & \text { LSTM-EWT } & \text { Altan et al. [89] }\end{array}$

\subsubsection{Hybrid machine learning methods}

Hybrid models combine machine learning models or combine a machine learning model with an optimization model to improve the predictivity of the machine learning model. Hybrid machine learning models are frequently employed among the reviewed articles in this study. Table 10 illustrates that 7 hybrid machine learning models are mainly proposed to stock price predictions (i.e. 4 out of 7) and three models have been developed to address problems related to e-commerce, namely order arrival prediction, dynamic credit risk evaluation, and product recommendation.

Table 10. List of hybrid machine learning models employed in Economic related fields

\begin{tabular}{ccc}
\hline Application & The hybrid method & Source \\
\hline & GA-SVM & Shekhar and Varshney [66] \\
Stock Market & ICA-SVM & Ahmadi et al. [67] \\
& GA-ANN & Ebadati and Mortazavi [68] \\
& GARCH-SVM & Johari et al. [69] \\
& AR-ANFIS & Leung et al. [81] \\
E-commerce & DT-ANN & Xu et al. [84] \\
& PCA- t-SNE-SVM & Saravanan and Charanya [85] \\
\hline
\end{tabular}

\subsubsection{Ensemble machine learning algorithms}

Ensemble machine learning algorithms or ensemble learning (EL) models use multiple learning algorithms to improve training processes and boost learning from data [6]. There is much evidence in the literature that ensemble classifiers and ensemble learning systems have been very effective in financial time series data. For instance, Rajesh et al. [51] combine RF, SVM, and K-neighbors classifiers, Weng et al. [56] used neural network regression ensemble, and Faghihi-Nezhad and Minaei-Bidgoli [62] applied an ANN-EL to predict stock values. Besides, Chen et al. [91], Lin et al. [92], Lahmiri et al. [93] respectively applied Bagged-pSVM and Boosted-pSVM models, a genetic algorithm with the naïve Bayes and SVM, SMOTE- AdaBoost- REP Tree to predict corporate bankruptcy. Ullah et al. [77] also took advantage of EL strategies and used an RF algorithm to predict the churn customers. Table 11 summers these studies which have used an ensemble model.

Table 11. List of ensemble models applied in the database on the current study

\begin{tabular}{ccc}
\hline Application & The ensemble method & Source \\
\hline \multirow{3}{*}{ Stock Market } & RF-SVM-K-neighbors & Rajesh et al. [51] \\
& NNRE & Weng et al. [56] \\
& ANN-EL & Faghihi-Nezhad and Minaei-Bidgoli \\
Corporate & Bagged-pSVM and Boosted- & [62] \\
Bankruptcy & Genetic Algorithm with the & Chen et al. [91] \\
& Naïve Bayes and SVM & Lin et al. [92] \\
& SMOTE- AdaBoost- REP Tree & Lahmiri et al. [93] \\
Marketing & RF - DNN & Ladyżyński et al. [76] \\
& RF & Ullah et al. [77]
\end{tabular}




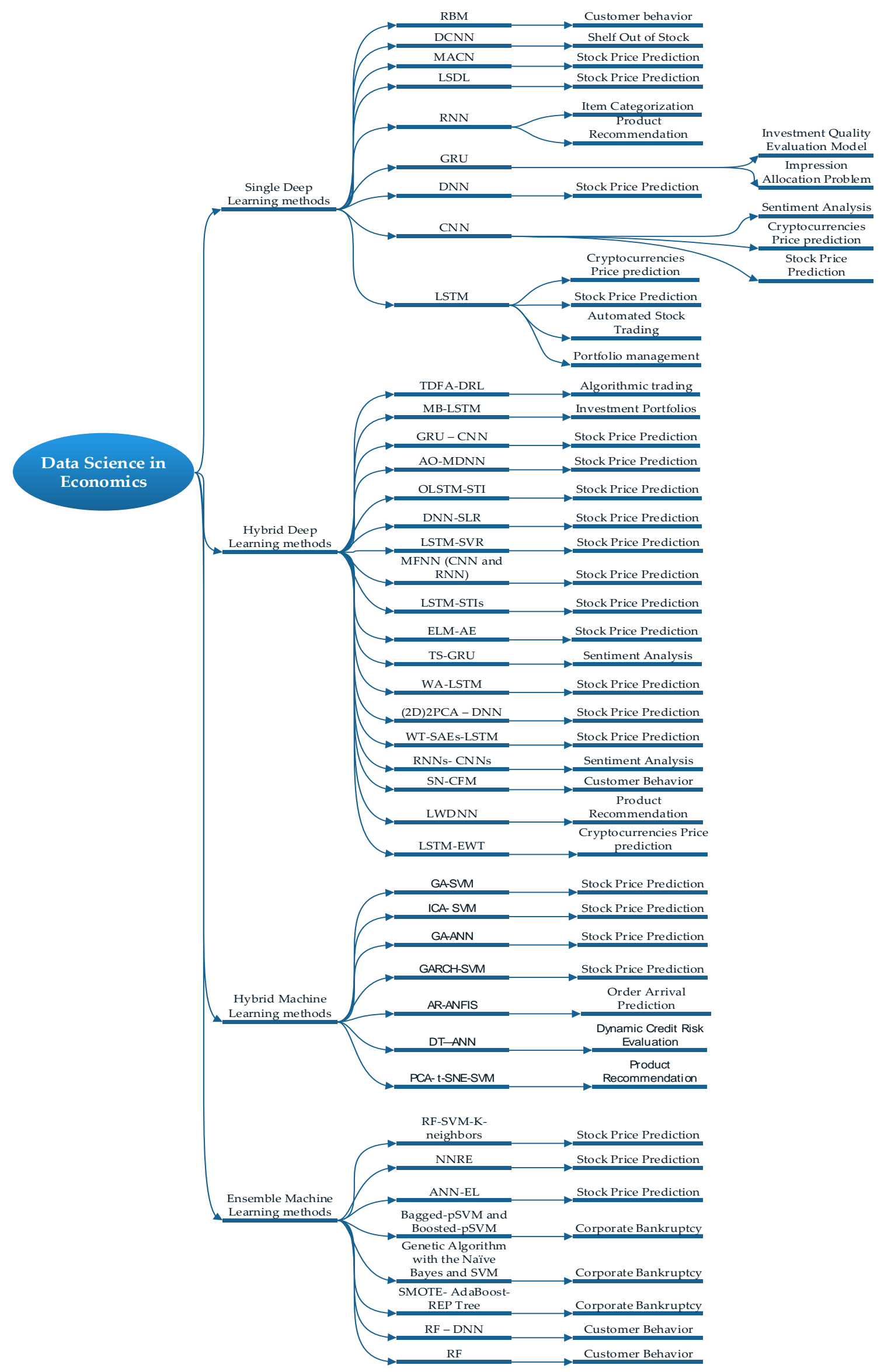

Figure 7. Taxonomy of data science advancements in Economics 


\subsection{Discussions on the taxonomy of data science advancements in Economics}

Oláh et al. [97] provided a checklist of items needed to validate the findings of the Prisma method. They argue that the articles selection should be explained in detail. Then, the characteristics of each article, including data source, data analysis method, as well as the findings of each study, should be written and referenced to that article. In the present study, first the processes of database formation of the study were described in detail in the methodology section and its processes were shown in Figure 2. Besides, the characteristics and results of each reviewed article are presented in detail in Tables 2-11. One of the contributions of this study is presenting the taxonomy of data science methods and applications in economics. Indeed, the output of reviewing the literature is presented in Figure 7. This study presents the advancement of deep learning models, hybrid deep learning models, hybrid machine learning models, and ensemble machine learning models in economics. It is figured out that overall, 42 unique algorithms employed among 57 reviewed articles in which 9 of them used single DL models (see Table 8), 18 hybrid DL models (see Table 9), 7 hybrid ML (see Table 10), and 8 ensemble models (see table 11). It has also been revealed that these 42 models have been used for different purposes, including algorithmic trading, automated stock trading, bankruptcy prediction, cryptocurrencies price prediction, customer behavior, shelf out of stock, impression allocation problem, investment quality evaluation model, item categorization, portfolio management, product recommendation, sentiment analysis, socially responsible investment portfolios, stock price prediction. It is also disclosed that data science algorithms mainly applied for financial time series data to forecast stock prices in which the LSTM model has been the most popular model for analyzing financial time series. CNN and DNN have been respectively the most applied algorithms among the reviewed articles in this study.

Twenty-five models out of 42 models presented the taxonomy are hybrid models that show the increasing trend of using hybrid models in the economy. As the hybrid models often outperform the single DL models, the popularity of hybridization of the methods have been increasing. The model performance techniques using the evaluation metrics, e.g., root-mean-squared error (RMSE) has been an standard strategy to evaluate the model performance in most cases. Therefore, the RMSE, which is among the most popular accuracy metrics of machine learning models, are summarized in Figure 8. The Lower the RMSE, the higher the accuracy of the model [10]. Figure 8 is a three-dimension graph in which the hybrid models are sorted in the $\mathrm{x}$ axis, the $\mathrm{y}$ axis represents the values of RMSE, and the $\mathrm{z}$ axis shows the number of times the models are used. According to Figure 8, the value of RMSE for the single DL models has been higher than the hybrid DL models that indicate the higher accuracy of hybrid DL models. This finding is consistent with the claims made in the literature (e.g. [9-11]) and it can also explain why the use of hybrid models has become trendy.

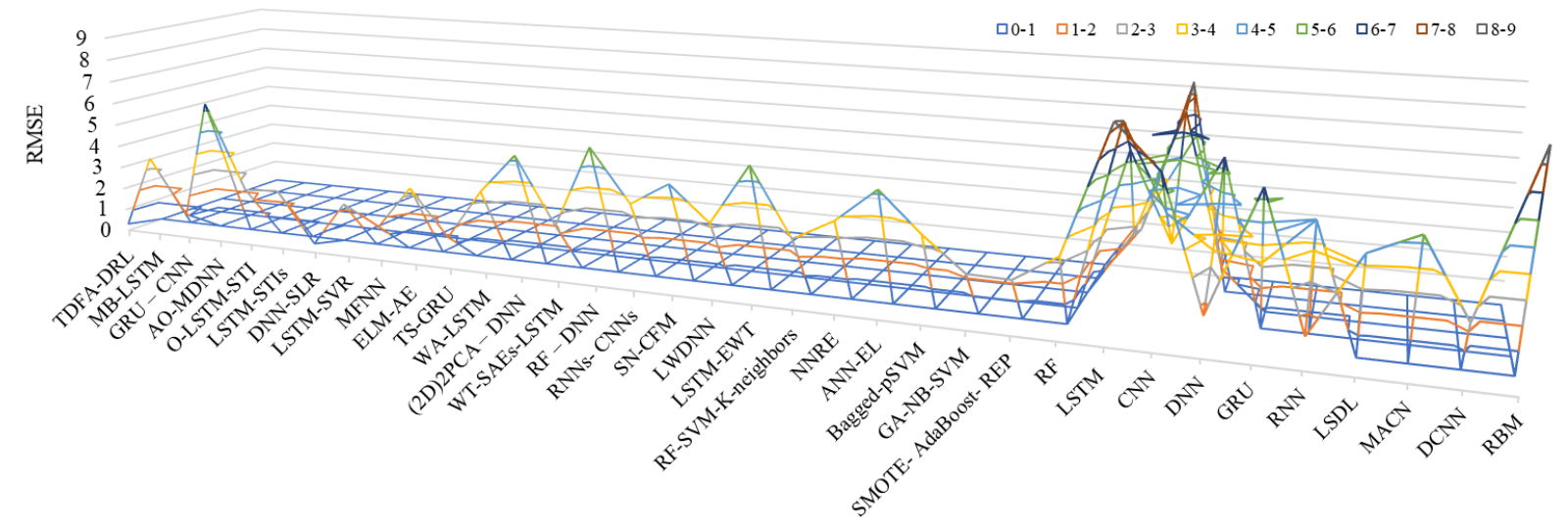

Figure 8. Comparison of root-mean-squared error values of hybrid deep learning models and deep learning 
The use of data science to address economics-related issues is increasing dramatically. Because data science has made significant advances in providing solutions to these problems. Providing the state-of-the-art of data science methods depicts a clear image of the advancements of data science in the economics-related fields. Therefore, the main objective of this study was to investigate the advances of data science in economics through a novel taxonomy of applications and methods. To that end, the Prisma method, a systematic literature review methodology, applied to survey all the publications indexed in the Scopus and Thomson Reuters Web-of-Science databases. The review is limited to published documents written in English and used data science techniques to solve problems in one of the economics-related fields. Finally, fifty-seven papers were selected for further consideration. The finding revealed that five economics-related fields, namely stock market, marketing, E-commerce, corporate banking, and cryptocurrency, utilized data science solutions. On the other hand, it was found that deep learning models, hybrid deep learning models, hybrid machine learning models, and ensemble machine learning models were used in each of these applications. It disclosed that the trends are on the advancement of hybrid models as 25 out of 42 models used among the reviewed articles have been hybrid models. According to Root-mean-square error accuracy metric, hybrid models had higher prediction accuracy than other algorithms, this can justify the prevalence of the use of hybrid models. The findings of this study are confined to these five applications related to the field of economics and cannot be generalized to other fields or disciplines. This research provides insights into the advancements of data science models and the application of these models in the field of economics, which provides guidance for researchers and practitioners in the field.

This research presents the state-of-the-art advancements in data science technologies and methods and their application in economics. The findings of this study provide guidance to researchers and practitioners in this field who, after familiarizing themselves with the latest developments in data science models, can select the appropriate model according to their application.

\section{Conclusion}

The use of data science techniques in economics and related fields is increasing day-by-day. The current study provides the latest developments in data science methods and their application in economics. The findings of this study show that the advancement of data science algorithms occurred in deep learning models, ensemble models, hybrid deep learning models, and hybrid machine learning models. Therefore, these four models were further investigated based on previous reports in five different areas: 1) stock market, in which stock price prediction was the main goal of most of the papers; 2) marketing, in which the objectives of the papers were mostly to study the customer behavior; 3) corporate bankruptcy; 4) cryptocurrency, which is a new trend in the economic field where researchers try to predict the price of digital money; and 5) e-commerce, in which the DS methods are applied mainly to increase the performance of e-commerce websites (e.g., by item categorization and product recommendation). This study elaborately provides the algorithms of deep learning models used in economics, data sourcing, and data science in each category. Findings reveal that the utilization of hybrid models has increased due to the higher prediction accuracy than single deep learning models. Specifically, LSTM, CNN, and DNN models have been the most applied models in literature to analyze financial time series data and predict stock price. Upon review of 57 articles in this work, it was found that all works employed 42 unique models, 25 of which were hybrid models, thus illustrating the trend of using such models in economics. The comparative results of RMSE values disclose that hybrid models show a remarkable low error level and, thus, higher prediction accuracy. Therefore, it is recommended to apply hybrid models to model and optimize objectives in all five fields studied in this research. Overall, this study provides state-of-the-art data science models in economics and generates insights for researchers and practitioners to determine the most appropriate model for their applications.

Author Contributions: Conceptualization, A.M.; investigation, S.N. and A.M.; writing-original draft preparation, S.N.; methodology, A.M.; writing - review and editing, S.N., A.M., P.D., P.G., F.F., S.S.B., U.R., J.G., 
A.H.G.; visualization, S.N; software, A.M.; project administration, A.M.; resources, A.M.; controlling the results, P.G, U.R., and A.H.G.; supervision, U.R., J.G., and A.H.G.; validation, A.M., U.R., J.G., and A.H.G. All authors have read and agreed to the published version of the manuscript.

Funding: This research is funded by the Alexander von Humboldt Foundation.

Acknowledgments: Support of the Alexander von Humboldt Foundation is acknowledged.

Conflicts of Interest: The authors declare no conflict of interest.

Appendix A

Acronyms

\begin{tabular}{ll}
\hline Acronym & Explanation \\
\hline (2D)2PCA & 2-Directional 2-Dimensional Principal Component Analysis \\
AE & Auto-Encoder \\
ANN & Artificial Neural Network \\
AO & Adam Optimizer \\
AR-ANFIS & Auto Regressive-Adaptive Neuro-Fuzzy Inference System \\
ARIMA & Autoregressive Integrated Moving Average Model \\
BRT & Boosted Regression Tree \\
CNN & Convolutional Neural Network \\
DCNN & Deep Convolutional Neural Network \\
DL & Deep Learning \\
DNN & Deep Neural Network \\
DNNC & Deep Neural Network Classifier \\
DRL & Deep Reinforcement Learning \\
DS & Data Science \\
DT-ANN & Decision Tree-Artificial Neural Network \\
EL & Ensemble Learning \\
ELM & Extreme Learning Machine \\
EWT & Empirical Wavelet Transform \\
GA-ANN & Genetic Algorithm-Artificial Neural Network \\
GARCH & Generalized Autoregressive Conditional Heteroskedasticity \\
GRDH & Group Method of Data Handling \\
GRNN & Generalized Regression Neural Networks \\
GRU & Gated Recurrent Unit \\
ICA & Imperialist Competition Algorithm \\
KNN & K-Nearest Neighbors \\
LOG & Logistic Regression Classifier \\
LSDL & Large-Scale Deep Learning \\
LSTM & Long Short-Term Memory \\
LWDNN & List-Wise Deep Neural Network \\
MACN & Multi-Agent Collaborated Network \\
MB-LSTM & Multivariate Bidirectional LSTM \\
MDNN & Multilayer Deep Neural Network \\
MFNN & Multi-Filters Neural Network \\
ML & Machine Learning \\
MLP & Multiple Layer Perceptron \\
\hline
\end{tabular}




\begin{tabular}{ll}
\hline NNRE & Neural Network Regression Ensemble \\
O-LSRM & Optimal Long Short-Term Memory \\
PCA & Principal Component Analysis \\
pSVM & Proportion Support Vector Machines \\
RBFNN & Radial Basis Function Neural Network \\
RBM & Restricted Boltzmann Machine \\
REP & Reduced Error Pruning \\
RF & Random Forest \\
RFR & Random Forest Regression \\
RNN & Recurrent Neural Network \\
SAE & Stacked Autoencoders \\
SLR & Stepwise Linear Regressions \\
SN-CFM & Similarity, Neighborhood-Based Collaborative Filtering Model \\
STI & Stock Technical Indicators \\
SVM & Support Vector Machine \\
SVR & Support Vector Regression \\
SVRE & Support Vector Regression Ensemble, \\
TDFA & Time-Driven Feature-Aware \\
TS-GRU & Two-Stream GRU \\
t-SNE & t-distributed stochastic neighbor embedding \\
WA & Wavelet Analysis \\
WT & Wavelet Transforms \\
K-NN & k-nearest neighbors algorithm \\
\hline
\end{tabular}

\section{References}

1. Salcedo-Sanz, S., Ghamisi, P., Piles, M., Werner, M., Cuadra, L., Moreno-Martínez, A., IzquierdoVerdiguier, E., Muñoz-Marí, J., Mosavi, A. and Camps-Valls, G., 2020. Machine learning information fusion in Earth observation: A comprehensive review of methods, applications and data sources. Information Fusion, 63, pp.256-272.

2. Mittal, S.; Stoean, C.; Kajdacsy-Balla, A.; Bhargava, R. Digital Assessment of Stained Breast Tissue Images for Comprehensive Tumor and Microenvironment Analysis. Frontiers in bioengineering and biotechnology 2019, 7, 246.

3. Stoean, R.; Iliescu, D.; Stoean, C. Segmentation of points of interest during fetal cardiac assesment in the first trimester from color Doppler ultrasound. arXiv preprint arXiv:1909.11903 2019.

4. Stoean, R.; Stoean, C.; Samide, A.; Joya, G. Convolutional Neural Network Learning Versus Traditional Segmentation for the Approximation of the Degree of Defective Surface in Titanium for Implantable Medical Devices. In Proceedings of International Work-Conference on Artificial Neural Networks; pp. 871-882.

5. Casalino, G.; Castellano, G.; Consiglio, A.; Liguori, M.; Nuzziello, N.; Primiceri, D. A Predictive Model for MicroRNA Expressions in Pediatric Multiple Sclerosis Detection. In Proceedings of International Conference on Modeling Decisions for Artificial Intelligence; pp. 177-188.

6. Mojrian, S., Pinter, G., Joloudari, J.H., Felde, I., Szabo-Gali, A., Nadai, L. and Mosavi, A., 2020, October. Hybrid Machine Learning Model of Extreme Learning Machine Radial basis function for Breast Cancer Detection and Diagnosis; a Multilayer Fuzzy Expert System. In 2020 RIVF International Conference on Computing and Communication Technologies (RIVF) (pp. 1-7). IEEE. 
7. Pinter, G., Felde, I., Mosavi, A., Ghamisi, P. and Gloaguen, R., 2020. COVID-19 Pandemic Prediction for Hungary; a Hybrid Machine Learning Approach. Mathematics, 8(6), p.890.

8. Torabi, M.; Hashemi, S.; Saybani, M.R.; Shamshirband, S.; Mosavi, A. A Hybrid clustering and classification technique for forecasting short-term energy consumption. Environmental Progress $\mathcal{E}$ Sustainable Energy 2019, 38, 66-76.

9. Mosavi, A.; Edalatifar, M. A hybrid neuro-fuzzy algorithm for prediction of reference evapotranspiration. In Proceedings of International Conference on Global Research and Education; pp. 235-243.

10. Mosavi, A.; Shamshirband, S.; Salwana, E.; Chau, K.-w.; Tah, J.H. Prediction of multi-inputs bubble column reactor using a novel hybrid model of computational fluid dynamics and machine learning. Engineering Applications of Computational Fluid Mechanics 2019, 13, 482-492.

11. Torabi, M.; Mosavi, A.; Ozturk, P.; Varkonyi-Koczy, A.; Istvan, V. A hybrid machine learning approach for daily prediction of solar radiation. In Proceedings of International Conference on Global Research and Education; pp. 266-274.

12. Lee, H.; Li, G.; Rai, A.; Chattopadhyay, A. Real-time anomaly detection framework using a support vector regression for the safety monitoring of commercial aircraft. Adv. Eng. Inf. 2020, 44, doi:10.1016/j.aei.2020.101071.

13. Husejinović, A. Credit card fraud detection using naive Bayesian and c4.5 decision tree classifiers. Period. eng. nat. sci. 2020, 8, 1-5.

14. Zhang, Y. Application of improved BP neural network based on e-commerce supply chain network data in the forecast of aquatic product export volume. Cogn. Sys. Res. 2019, 57, 228-235, doi:10.1016/j.cogsys.2018.10.025.

15. Sundar, G.; Satyanarayana, K. Multi layer feed forward neural network knowledge base to future stock market prediction. International Journal of Innovative Technology and Exploring Engineering 2019, 8, 10611075, doi:10.35940/ijitee.K1218.09811S19.

16. Hew, J.J.; Leong, L.Y.; Tan, G.W.H.; Ooi, K.B.; Lee, V.H. The age of mobile social commerce: An Artificial Neural Network analysis on its resistances. Technol. Forecast. Soc. Change 2019, 144, 311-324, doi:10.1016/j.techfore.2017.10.007.

17. Abdillah, Y.; Suharjito. Failure prediction of e-banking application system using Adaptive Neuro Fuzzy Inference System (ANFIS). Int. J. Electr. Comput. Eng. 2019, 9, 667-675, doi:10.11591/ijece.v9i1.pp.667675.

18. Sabaitytè, J.; Davidavičienè, V.; Straková, J.; Raudeliūnienè, J. Decision tree modelling of E-consumers' preferences for internet marketing communication tools during browsing. E M Ekon. Manage. 2019, 22, 206-224, doi:10.15240/tul/001/2019-1-014.

19. Zatevakhina, A.; Dedyukhina, N.; Klioutchnikov, O. Recommender Systems-The Foundation of an Intelligent Financial Platform: Prospects of Development. In Proceedings of 2019 International Conference on Artificial Intelligence: Applications and Innovations (IC-AIAI); pp. 104-1046.

20. Benlahbib, A.; Nfaoui, E.H. A hybrid approach for generating reputation based on opinions fusion and sentiment analysis. Journal of Organizational Computing and Electronic Commerce 2020, 30, 9-27.

21. Fujiyoshi, H.; Hirakawa, T.; Yamashita, T. Deep learning-based image recognition for autonomous driving. IATSS Research 2019.

22. Mathis, M.W.; Mathis, A. Deep learning tools for the measurement of animal behavior in neuroscience. Current Opinion in Neurobiology 2020, 60, 1-11. 
23. Wang, H.; Lei, Z.; Zhang, X.; Zhou, B.; Peng, J. A review of deep learning for renewable energy forecasting. Energy Conversion and Management 2019, 198, 111799.

24. Durairaj, M.; Krishna Mohan, B.H. A review of two decades of deep learning hybrids for financial time series prediction. International Journal on Emerging Technologies 2019, 10, 324-331.

25. Guermoui, M.; Melgani, F.; Gairaa, K.; Mekhalfi, M.L. A comprehensive review of hybrid models for solar radiation forecasting. Journal of Cleaner Production 2020, 120357.

26. Pradeepkumar, D.; Ravi, V. Soft computing hybrids for FOREX rate prediction: A comprehensive review. Computers \& Operations Research 2018, 99, 262-284.

27. Hosni, M.; Abnane, I.; Idri, A.; de Gea, J.M.C.; Alemán, J.L.F. Reviewing ensemble classification methods in breast cancer. Computer methods and programs in biomedicine 2019.

28. Song, X.; Jiao, L.; Yang, S.; Zhang, X.; Shang, F. Sparse coding and classifier ensemble based multiinstance learning for image categorization. Signal processing 2013, 93, 1-11.

29. Chung, Y.-W.; Khaki, B.; Li, T.; Chu, C.; Gadh, R. Ensemble machine learning-based algorithm for electric vehicle user behavior prediction. Applied Energy 2019, 254, 113732.

30. AlKandari, M.; Ahmad, I. Solar Power Generation Forecasting Using Ensemble Approach Based on Deep Learning and Statistical Methods. Applied Computing and Informatics 2019.

31. Liberati, A.; Altman, D.G.; Tetzlaff, J.; Mulrow, C.; Gøtzsche, P.C.; Ioannidis, J.P.; Clarke, M.; Devereaux, P.J.; Kleijnen, J.; Moher, D. The PRISMA statement for reporting systematic reviews and meta-analyses of studies that evaluate health care interventions: explanation and elaboration. Journal of clinical epidemiology 2009, 62, e1-e34.

32. Moon, K.S.; Kim, H. Performance of deep learning in prediction of stock market volatility. Economic Computation and Economic Cybernetics Studies and Research 2019, 53, 77-92, doi:10.24818/18423264/53.2.19.05.

33. Fischer, T.; Krauss, C. Deep learning with long short-term memory networks for financial market predictions. European Journal of Operational Research 2018, 270, 654-669, doi:10.1016/j.ejor.2017.11.054.

34. Tamura, K.; Uenoyama, K.; Iitsuka, S.; Matsuo, Y. Model for evaluation of stock values by ensemble model using deep learning. Transactions of the Japanese Society for Artificial Intelligence 2018, 33, doi:10.1527/tjsai.A-H51.

35. Wang, W.; Li, W.; Zhang, N.; Liu, K. Portfolio formation with preselection using deep learning from long-term financial data. Expert Systems with Applications 2020, 143, 113042.

36. Fister, D.; Mun, J.C.; Jagric, V.; Jagric, T. Deep learning for stock market trading: A superior trading strategy? Neural Network World 2019, 29, 151-171, doi:10.14311/NNW.2019.29.011.

37. Stoean, C.; Paja, W.; Stoean, R.; Sandita, A. Deep architectures for long-term stock price prediction with a heuristic-based strategy for trading simulations. PloS one 2019, 14.

38. Agrawal, M.; Khan, A.U.; Shukla, P.K. Stock price prediction using technical indicators: A predictive model using optimal deep learning. International Journal of Recent Technology and Engineering 2019, 8, 2297-2305, doi:10.35940/ijrteB3048.078219.

39. Bao, W.; Yue, J.; Rao, Y. A deep learning framework for financial time series using stacked autoencoders and long-short term memory. PLOS ONE 2017, 12, doi:10.1371/journal.pone.0180944.

40. Yan, H.; Ouyang, H. Financial Time Series Prediction Based on Deep Learning. Wireless Personal Communications 2018, 102, 683-700, doi:10.1007/s11277-017-5086-2.

41. Vo, N.N.; He, X.; Liu, S.; Xu, G. Deep learning for decision making and the optimization of socially responsible investments and portfolio. Decision Support Systems 2019, 124, 113097. 
42. Fang, Y.; Chen, J.; Xue, Z. Research on quantitative investment strategies based on deep learning. Algorithms 2019, 12, doi:10.3390/a12020035.

43. Lei, K.; Zhang, B.; Li, Y.; Yang, M.; Shen, Y. Time-driven feature-aware jointly deep reinforcement learning for financial signal representation and algorithmic trading. Expert Systems with Applications 2020, 140, doi:10.1016/j.eswa.2019.112872.

44. Sabeena, J.; Venkata Subba Reddy, P. A modified deep learning enthused adversarial network model to predict financial fluctuations in stock market. International Journal of Engineering and Advanced Technology 2019, 8, 2996-3000, doi:10.35940/ijeat.F9011.088619.

45. Das, S.; Mishra, S. Advanced deep learning framework for stock value prediction. International Journal of Innovative Technology and Exploring Engineering 2019, 8, 2358-2367, doi:10.35940/ijitee.B2453.0881019.

46. Go, Y.H.; Hong, J.K. Prediction of stock value using pattern matching algorithm based on deep learning. International Journal of Recent Technology and Engineering 2019, 8, 31-35, doi:10.35940/ijrte.B1007.0782S619.

47. Gonçalves, R.; Ribeiro, V.M.; Pereira, F.L.; Rocha, A.P. Deep learning in exchange markets. Information Economics and Policy 2019, 47, 38-51, doi:10.1016/j.infoecopol.2019.05.002.

48. Moews, B.; Herrmann, J.M.; Ibikunle, G. Lagged correlation-based deep learning for directional trend change prediction in financial time series. Expert Systems with Applications 2019, 120, 197-206, doi:10.1016/j.eswa.2018.11.027.

49. Song, Y.; Lee, J.W.; Lee, J. A study on novel filtering and relationship between input-features and targetvectors in a deep learning model for stock price prediction. Applied Intelligence 2019, 49, 897-911, doi:10.1007/s10489-018-1308-x.

50. Long, W.; Lu, Z.; Cui, L. Deep learning-based feature engineering for stock price movement prediction. Knowledge-Based Systems 2019, 164, 163-173, doi:10.1016/j.knosys.2018.10.034.

51. Rajesh, P.; Srinivas, N.; Vamshikrishna Reddy, K.; VamsiPriya, G.; Vakula Dwija, M.; Himaja, D. Stock trend prediction using Ensemble learning techniques in python. International Journal of Innovative Technology and Exploring Engineering 2019, 8, 150-155.

52. Sim, H.S.; Kim, H.I.; Ahn, J.J. Is Deep Learning for Image Recognition Applicable to Stock Market Prediction? Complexity 2019, 2019, doi:10.1155/2019/4324878.

53. Agrawal, M.; Khan, A.U.; Shukla, P.K. Stock indices price prediction based on technical indicators using deep learning model. International Journal on Emerging Technologies 2019, 10, 186-194.

54. Tashiro, D.; Matsushima, H.; Izumi, K.; Sakaji, H. Encoding of high-frequency order information and prediction of short-term stock price by deep learning. Quantitative Finance 2019, 19, 1499-1506, doi:10.1080/14697688.2019.1622314.

55. Sirignano, J.; Cont, R. Universal features of price formation in financial markets: perspectives from deep learning. Quantitative Finance 2019, 19, 1449-1459, doi:10.1080/14697688.2019.1622295.

56. Weng, B.; Lu, L.; Wang, X.; Megahed, F.M.; Martinez, W. Predicting short-term stock prices using ensemble methods and online data sources. Expert Systems with Applications 2018, 112, 258-273.

57. Preeti; Dagar, A.; Bala, R.; Singh, R.P. Financial time series forecasting using deep learning network. In Communications in Computer and Information Science, Springer Verlag: 2019; Vol. 899, pp 23-33.

58. Sohangir, S.; Wang, D.; Pomeranets, A.; Khoshgoftaar, T.M. Big Data: Deep Learning for financial sentiment analysis. Journal of Big Data 2018, 5, doi:10.1186/s40537-017-0111-6. 
59. Lien Minh, D.; Sadeghi-Niaraki, A.; Huy, H.D.; Min, K.; Moon, H. Deep learning approach for shortterm stock trends prediction based on two-stream gated recurrent unit network. IEEE Access 2018, 6, 55392-55404, doi:10.1109/ACCESS.2018.2868970.

60. Das, S.R.; Mokashi, K.; Culkin, R. Are markets truly efficient? Experiments using deep learning algorithms for market movement prediction. Algorithms 2018, 11, doi:10.3390/a11090138.

61. Kim, J.J.; Cha, S.H.; Cho, K.H.; Ryu, M. Deep reinforcement learning based multi-agent collaborated network for distributed stock trading. International Journal of Grid and Distributed Computing 2018, 11, 11-20, doi:10.14257/ijgdc.2018.11.2.02.

62. Faghihi-Nezhad, M.-T.; Minaei-Bidgoli, B. Prediction of Stock Market Using an Ensemble Learningbased Intelligent Model. Industrial Engineering \& Management Systems 2018, 17, 479-496.

63. Chong, E.; Han, C.; Park, F.C. Deep learning networks for stock market analysis and prediction: Methodology, data representations, and case studies. Expert Systems with Applications 2017, 83, 187-205, doi:10.1016/j.eswa.2017.04.030.

64. Dingli, A.; Fournier, K.S. Financial time series forecasting - a deep learning approach. International Journal of Machine Learning and Computing 2017, 7, 118-122, doi:10.18178/ijmlc.2017.7.5.632.

65. Singh, R.; Srivastava, S. Stock prediction using deep learning. Multimedia Tools and Applications 2017, 76, 18569-18584, doi:10.1007/s11042-016-4159-7.

66. Shekhar, S.; Varshney, N. A hybrid GA-SVM and sentiment analysis for forecasting stock market movement direction. Test Eng Manage 2020, 82, 64-72.

67. Ahmadi, E.; Jasemi, M.; Monplaisir, L.; Nabavi, M.A.; Mahmoodi, A.; Amini Jam, P. New efficient hybrid candlestick technical analysis model for stock market timing on the basis of the Support Vector Machine and Heuristic Algorithms of Imperialist Competition and Genetic. Expert Systems with Applications 2018, 94, 21-31, doi:10.1016/j.eswa.2017.10.023.

68. Ebadati, O.M.E.; Mortazavi, M.T. An efficient hybrid machine learning method for time series stock market forecasting. Neural Network World 2018, 28, 41-55, doi:10.14311/NNW.2018.28.003.

69. Johari, S.N.M.; Farid, F.H.M.; Nasrudin, N.A.E.B.; Bistamam, N.S.L.; Shuhaili, N.S.S.M. Predicting stock market index using hybrid intelligence model. International Journal of Engineering and Technology(UAE) 2018, 7, 36-39, doi:10.14419/ijet.v7i3.15.17403.

70. Ghamisi, P.; Höfle, B.; Zhu, X.X. Hyperspectral and LiDAR data fusion using extinction profiles and deep convolutional neural network. IEEE Journal of Selected Topics in Applied Earth Observations and Remote Sensing 2016, 10, 3011-3024.

71. Chen, Y.; Jiang, H.; Li, C.; Jia, X.; Ghamisi, P. Deep feature extraction and classification of hyperspectral images based on convolutional neural networks. IEEE Transactions on Geoscience and Remote Sensing 2016, 54, 6232-6251.

72. Duan, P.; Kang, X.; Li, S.; Ghamisi, P. Multichannel Pulse-Coupled Neural Network-Based Hyperspectral Image Visualization. IEEE Transactions on Geoscience and Remote Sensing 2019.

73. Li, S.; Song, W.; Fang, L.; Chen, Y.; Ghamisi, P.; Benediktsson, J.A. Deep learning for hyperspectral image classification: An overview. IEEE Transactions on Geoscience and Remote Sensing 2019, 57, 66906709.

74. Paolanti, M.; Romeo, L.; Martini, M.; Mancini, A.; Frontoni, E.; Zingaretti, P. Robotic retail surveying by deep learning visual and textual data. Robotics and Autonomous Systems 2019, 118, 179-188, doi:10.1016/j.robot.2019.01.021. 
75. Dingli, A.; Marmara, V.; Fournier, N.S. Comparison of deep learning algorithms to predict customer churn within a local retail industry. International Journal of Machine Learning and Computing 2017, 7, 128132, doi:10.18178/ijmlc.2017.7.5.634.

76. $\quad$ Ładyżyński, P.; Żbikowski, K.; Gawrysiak, P. Direct marketing campaigns in retail banking with the use of deep learning and random forests. Expert Systems with Applications 2019, 134, 28-35, doi:10.1016/j.eswa.2019.05.020.

77. Ullah, I.; Raza, B.; Malik, A.K.; Imran, M.; Islam, S.U.; Kim, S.W. A churn prediction model using random forest: analysis of machine learning techniques for churn prediction and factor identification in telecom sector. IEEE Access 2019, 7, 60134-60149.

78. Agarwal, S. Deep Learning-based Sentiment Analysis: Establishing Customer Dimension as the Lifeblood of Business Management. Global Business Review 2019, 10.1177/0972150919845160, doi:10.1177/0972150919845160.

79. Shamshirband, S.; Khader, J.; Gani, S. Predicting consumer preferences in electronic market based on IoT and Social Networks using deep learning based collaborative filtering techniques. Electronic Commerce Research 2019, 10.1007/s10660-019-09377-0, doi:10.1007/s10660-019-09377-0.

80. Lei, Z. Research and analysis of deep learning algorithms for investment decision support model in electronic commerce. Electronic Commerce Research 2019, 10.1007/s10660-019-09389-w, doi:10.1007/s10660-019-09389-w.

81. Leung, K.H.; Choy, K.L.; Ho, G.T.S.; Lee, C.K.M.; Lam, H.Y.; Luk, C.C. Prediction of B2C e-commerce order arrival using hybrid autoregressive-adaptive neuro-fuzzy inference system (AR-ANFIS) for managing fluctuation of throughput in e-fulfilment centres. Expert Systems with Applications 2019, 134, 304-324, doi:10.1016/j.eswa.2019.05.027.

82. Cai, Q.; Filos-Ratsikas, A.; Tang, P.; Zhang, Y. Reinforcement Mechanism Design for e-commerce. In Proceedings of Proceedings of the 2018 World Wide Web Conference; pp. 1339-1348.

83. Ha, J.-W.; Pyo, H.; Kim, J. Large-scale item categorization in e-commerce using multiple recurrent neural networks. In Proceedings of Proceedings of the 22nd ACM SIGKDD International Conference on Knowledge Discovery and Data Mining; pp. 107-115.

84. Xu, Y.Z.; Zhang, J.L.; Hua, Y.; Wang, L.Y. Dynamic credit risk evaluation method for e-commerce sellers based on a hybrid artificial intelligence model. Sustainability (Switzerland) 2019, 11, doi:10.3390/su11195521.

85. Saravanan, V.; Sathya Charanya, C. E-commerce product classification using lexical based hybrid feature extraction and SVM. International Journal of Innovative Technology and Exploring Engineering 2019, 9, 1885-1891, doi:10.35940/ijitee.L3608.119119.

86. Wang, Y.; Mo, D.Y.; Tseng, M.M. Mapping customer needs to design parameters in the front end of product design by applying deep learning. CIRP Annals 2018, 67, 145-148.

87. Wu, C.; Yan, M. Session-aware information embedding for e-commerce product recommendation. In Proceedings of Proceedings of the 2017 ACM on conference on information and knowledge management; pp. 2379-2382.

88. Lahmiri, S.; Bekiros, S. Cryptocurrency forecasting with deep learning chaotic neural networks. Chaos, Solitons and Fractals 2019, 118, 35-40, doi:10.1016/j.chaos.2018.11.014.

89. Altan, A.; Karasu, S.; Bekiros, S. Digital currency forecasting with chaotic meta-heuristic bio-inspired signal processing techniques. Chaos, Solitons $\mathcal{E}$ Fractals 2019, 126, 325-336. 
90. Jiang, Z.; Liang, J. Cryptocurrency portfolio management with deep reinforcement learning. In Proceedings of 2017 Intelligent Systems Conference (IntelliSys); pp. 905-913.

91. Chen, Z.; Chen, W.; Shi, Y. Ensemble learning with label proportions for bankruptcy prediction. Expert Systems with Applications 2020, 146, 113155.

92. Lin, W.C.; Lu, Y.H.; Tsai, C.F. Feature selection in single and ensemble learning-based bankruptcy prediction models. Expert Systems 2019, 36, e12335.

93. Lahmiri, S.; Bekiros, S.; Giakoumelou, A.; Bezzina, F. Performance assessment of ensemble learning systems in financial data classification. Intelligent Systems in Accounting, Finance and Management 2020, https://doi.org/10.1002/isaf.1460, 1-7, doi:https://doi.org/10.1002/isaf.1460.

94. Faris, H.; Abukhurma, R.; Almanaseer, W.; Saadeh, M.; Mora, A.M.; Castillo, P.A.; Aljarah, I. Improving financial bankruptcy prediction in a highly imbalanced class distribution using oversampling and ensemble learning: a case from the Spanish market. Progress in Artificial Intelligence 2019, 1-23.

95. Hinton, G.E.; Salakhutdinov, R.R. Reducing the dimensionality of data with neural networks. science 2006, 313, 504-507.

96. Zhang, Q.; Yang, L.T.; Chen, Z.; Li, P. A survey on deep learning for big data. Information Fusion 2018, 42, 146-157.

97. Oláh, J.; Krisán, E.; Kiss, A.; Lakner, Z.; Popp, J. PRISMA Statement for Reporting Literature Searches in Systematic Reviews of the Bioethanol Sector. Energies 2020, 13, 2323. 